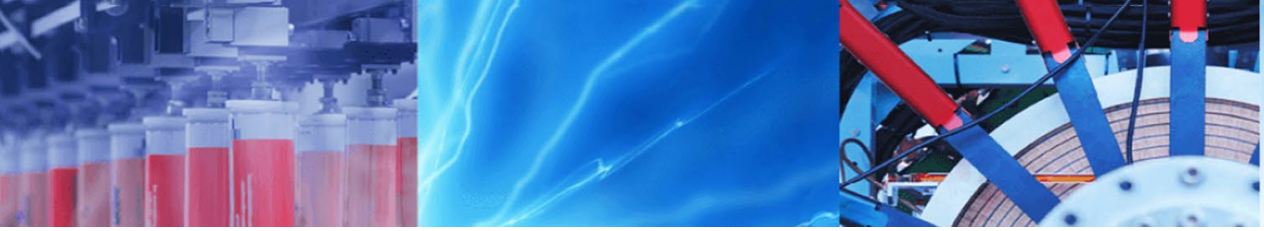

Research Article

\title{
Adaptive opto-electromechanical silicon-on-insulator increased bandwidth accelerometer
}

\author{
Ali Abdulsattar Hussein ${ }^{1}$ (I)
}

Received: 21 May 2021 / Accepted: 13 December 2021

Published online: 04 January 2022

(c) The Author(s) 2021 OPEN

\begin{abstract}
This paper studies the construction of a compact one-dimension-sensing iscreased bandwidth photonic accelerometer using cascaded groups of continued sections of a $50 \mathrm{ng}$ seismic mass each attached to the silicon beams of two under etched slot waveguide electrostatic phase shift elements acting as voltage-controlled adaptive-precision springs. The accelerometer sensitivity is shown to be significantly increased by applying equal electrode voltages. Simulation results indicate that the sensitivity dynamic range is about $76 \mathrm{~dB}$ combining both open-loop and closed-loop voltage control of the sensor. The operation bandwidth of the accelerometer may be increased up to $250 \mathrm{kHz}$ due to the cascaded multi-section architecture of the sensor. This advantage gives significant relief to the limitation in bandwidth response of single section counterparts. The sensor may be designed to detect impact accelerations up to $10^{4} \mathrm{~ms}^{-2}$ and yet can still be electrostatically driven to detect sub-gravitational accelerations. The application of negative feedback voltage control to hold the seismic mass at close distances from a standstill is shown to significantly increase the acceleration detection range. The construction uses all in-plane components based on a silicon-on-insulator template with $300 \mathrm{~nm}$ of silicon core thickness. The proposed electromechanical suspension system and the electric feeding arrangements are the most simple. The accelerometer performance is theoretically deterministic. The study is based on performing numerical analysis for the electromechanical suspension system. The waveguides are simulated utilizing the VPIphotonics industry standard. Applications may include the automobile and aerospace industries, underwater sonar, industrial ultrasonic detection, seismology predictions, and medical ultrasonography.
\end{abstract}

\section{Article Highlights}

- The cascading of compact high-speed accelerometer sections allows increasing the bandwidth response of the proposed sensor by many folds compared to its single-mass single-section counterparts.
- The suspension structure is electrostatically controlled by two voltages enabling widely controlling the sensitivity and detection range of the accelerometer.

- The proposed accelerometer may fit wide applications achieving high detection speeds and super sensitivities utilizing a small footprint and power-efficient structure.

Keywords Photonic sensor - Increased bandwidth accelerometer - Adaptive sensor - Opto-electromechanical sensor . Mach-Zehnder interferometer · Suspended slot waveguide

Ali Abdulsattar Hussein, ahussein@georgebrown.ca| 'School of Engineering Technologies and Architectural Studies, George Brown College, Toronto, ON M5T 2T9, Canada. 


\section{Introduction}

There has been growing interest in contemporary literature to miniaturize displacement-based sensors for the measurement of physical quantities like pressure, strain, acceleration, hypersonic speeds, nano displacement vibrations, gravitational orientation, angular momentums, and acoustic ultrasound waves. The miniaturization of displacement-type sensors for lightweight and small size merits is desirable for applications in portable customer electronic devices, immersive virtual reality technology with precise motion tracking control, flying objects like drones, the prospective autonomous transportation industry, industrial process sensors, inertial navigation, and medical ultrasound imaging. The miniaturization of sensors provides advantages of small footprint, lightweight, efficient power consumption, chip integration with other electronic devices, and improved performance of faster operation speed, higher sensitivity, and increased resolution. Microelectromechanical systems (MEMS) and nanoelectromechanical systems (NEMS) are standard technologies used in constructing miniaturized sensors to measure pressure, acceleration, sub-nano displacement, strain, and torsion. The principles of operation of these electromechanical sensors rely on either capacitive effect, piezoelectric effect, or photonic effect. Capacitive MEMS reported extensively in the literature, e.g. [1-4], offer the advantages of low-temperature dependence, low-cost technology, and simple detection methods but the required large $\mathrm{pF}$ range capacitance puts a limit on miniaturizing the sensor size. Also, the stray capacitance of the electric feeding arrangements in capacitive MEMS results in smaller figures of sensitivity and resolution compared to photonic sensors. Albeit piezoelectric sensors, e.g. [5-8], are attractive for their low cost and simple detection method they are not accurate as their photonic counterparts. Many of the piezoelectric sensors with a high stiffness-to-mass ratio of their vibratory structure fit well for the generation and detection of high-frequency acoustic waves in the $\mathrm{MHz}$ range, and hence they are traditionally used in ultrasonography. The less sensitive and lower accuracy capacitive MEMS cover the operation at lower frequencies where the piezoelectric effect of highly ridge structures fails. Another issue associated with capacitive MEMS is that the provision of feedback voltage to hold the test mass (also called proof mass, seismic mass, or actuator mass) at the reset position requires using an additional electrically isolated electrostatic structure which would increase the footprint of the sensor. This difficulty in providing feedback control voltage is because both the test capacitance and the electrostatic realignment capacitance use the same type of electric signal.
Photonic sensors, e.g. [9-12], can provide better accuracy and higher sensitivity levels than their capacitive and piezoelectric counterparts albeit they are based on more expensive technology. The optical readout sensing methods continued dominating the attention in recent years for various applications, e.g. [13-16], for their high performance and immunity against electromagnetic interference (EMI). The noise cancelation circuit needs to be placed close to the capacitive or piezoelectric sensor itself to reduce the EMI effect resulting in increasing the bulk size of the sensor probe. The silicon-on-insulator (SOI) photonic sensors provide the advantage of naturally separating the electric feedback voltage used to control the positioning of the test mass from the photonic signal used in measuring the physical test quantity such as acceleration allowing reducing the footprint of the sensor to standard photonic chip foundries. The contemporary nanomachining technology with deep under-etching in the box $\left(\mathrm{SiO}_{2}\right)$ layer allows the construction of SOI-based accelerometers as can be found in the work of Zandi et al. [10] which utilized a Fabry-Pérot optical cavity with strip waveguide and distributed Bragg reflector (DBR) mirrors. The silicon (Si) springs of the accelerometer of Zandi et al. used to anchor the proof mass make it not easily possible to support providing feedback electrostatic positioning force. The work of Westerveld et al. [11] reported the use of SOI-based ring resonator as a strain gauge for ultrasound detection achieving sensitivity levels compared to those of traditional piezoelectric imaging devices. The elongation of the racetrack length of the ring resonator of Westerveld et al. due to the applied strain induces a change in the effective index of the waveguide and detunes the ring waveguide resonant frequency. However, such a strain gauge requires special mechanical arrangements to magnify the strain applied to a thinned spot of the SOI chip addressing the fabrication process as complicated and expensive. The work of Miao et al. [17] reported a highquality cavity optomechanical accelerometer provided with feedback voltage to electrostatically control the optomechanical system. However, the accelerometer of Miao et al. used out-of-plane thick silicon (Si) microdisk optical resonator to detect the out-of-plane mechanical motion and used an overall complicated anchoring structure that needs out-of-plane flexible insulating attachments making it expensive and challenging to NanoFABs that run standard SOI fabrication procedures. In summary, the above surveyed SOI-based accelerometers either use complicated anchoring arrangements for the proof mass or do not include voltage-controlled actuation of the mechanical system and are hard to fit different applications. Therefore, it becomes evident that there is a need for the design of a photonic accelerometer that uses in-plane components 
with a simple suspension structure providing adaptive voltage-controlled performance scalable to different applications and covering a wide band of frequencies.

Different optical detection principles to sense the motion have been proposed in the literature to either modulate the intensity or phase of an optical signal or wavelength modulates the resonance characteristics of optical filters. Accelerometers based on directly modulating the intensity of an optical carrier [18-20] advantage simple structure, low fabrication cost, simple detection electronics, and the need for a simple optical source but they result in low detection quality for being affected by the fluctuation in the optical source intensity. Ahmadian et al. [21] proposed a graphene-based optical MEMS accelerometer that depends on the intensity modulation of the light. The accelerometer of Ahmadian et al. utilizes the light absorption properties of a multi-layer graphene finger placed within a gap between two optical waveguides to modulate the intensity of the light. The suspension system of the sensor uses serpentine springs. It also uses stoppers to restrict the motion of the proof mass in the out-of-plane direction. By adjusting the length of the graphene finger to fit half the width of the gap between the two optical waveguides at standstill the sensor is capable of measuring the acceleration in opposite directions along the axis of interest. The sensor advantages the simple structure, low cost, and high sensitivity, but its first resonance frequency was limited at $15.5 \mathrm{kHz}$ and the available bandwidth is lower. It is also affected like other intensity-based accelerometers by the intensity noise of the optical source.

Sheikhaleh et al. [15] proposed a microelectromechanical accelerometer using a two-dimensional photonic crystal add-drop filter that relies on the wavelength modulation approach of the characteristics of a ring resonator. The sensor of Sheikhaleh et al. is based on detecting the central wavelength shift of the optical resonant mode due to modifying the radius of a ring resonator structure driven by the displacement of the proof mass due to the applied acceleration. The accelerometer of Sheikhaleh et al. is compact with only $200 \times 200 \mu \mathrm{m}^{2}$ of the core area, SOl-based, and set to measure accelerations within the range of $\pm 156 \mathrm{~g}$. However, due to the nature of the serpentine spring, the suspension system of the single proof mass may exhibit different motion modes in all directions with different resonant frequencies. The reported bandwidth of the proposed sensor of Sheikhaleh et al. in the direction of interest was taken as $1 / 20$ of the first resonant frequency which was set at $12.935 \mathrm{kHz}$. This result of low bandwidth response exemplifies the expected drawback of single mass sensors as the management of the suspension system stiffness-to-mass ratio, response bandwidth, and sensitivity of the sensor cannot be all set to optimal values.

Zhao et al. [13] reported an optical MEMS Fabry-Pe'rot based accelerometer utilizing a G-shaped cantilever suspension structure. The principle of operation of the sensor is based on modulating the cavity length between two partially reflective plane mirrors. The proposed integrated sensing chip of the proposed sensor is composed of a Fabry-Pe'rot cavity, a proof mass, two G-shaped beams, and a base frame. The suspension spring of the sensor is affected by both torsion and bending forces in the direction of acceleration measurement. The low stiffness of the suspension system combined with the high accuracy of the optical Fabry-Pe'rot cavity resonance system allows designing the system to achieve high sensitivity and resolution figures of $183.793 \mathrm{~V} / \mathrm{g}$ and $300 \mathrm{ng}$, respectively, but at the expense of low detection speed of the sensor. The G-shaped suspension system may exhibit different mechanical vibration modes that may affect the measured acceleration and the reported first resonant frequency in the direction of interest is around $89 \mathrm{~Hz}$ providing that the available bandwidth is lower than this value.

Malayappan et al. [14] presented a waveguide Bragg grating-based quad-beam accelerometer that could achieve a high figure of sensitivity of $30 \mathrm{pm} / \mathrm{g}$. The proposed sensor consists of Si bulk micromachined proof mass suspended by four silica beams. Three sets of ridge gratings of non-overlapping Bragg wavelengths are positioned on two orthogonal suspension beams and the proof mass. The Bragg gratings act as guided-wave optical reflection filters. The accelerated proof mass of the sensor introduces compressional and tensile stress on the suspension amorphous-silicon beams. The deformation in the beams affects the optical characteristics of the gratings that are positioned on them and result in shifting the Bragg resonance wavelengths of those gratings. The resonance wavelength of the grating on the proof mass is not affected by the applied acceleration and serves as a reference for acceleration measurement and cancellation of noise due to temperature variation. The proposed strain-based accelerometer may be affected by the direction of the applied acceleration. It may also be affected by torsion forces adding uncertainty factors to realize the acceleration direction. The fundamental frequency of resonance of the sensor for the out-of-plane vibration was determined as $92.43 \mathrm{~Hz}$. The interrogation methods available to detect the shift in the Bragg resonance peaks for this sensor are costly. The sensor is also sensitive to temperature variations that affect its performance and requires suitable temperature compensation that would increase the expense of the detection circuity.

In summary, many of the photonic sensors found in the literature that are based on single element suspension 
architectures suffer when it comes to optimizing the requirement of achieving high sensitivity of the measured acceleration and the requirement of increasing the bandwidth response. On the other hand, maximizing the sensitivity of the accelerometer may not suit all kinds of applications and put limits on the usability of the high-sensitivity sensor. To provide a resolution for the aforementioned difficulties of many surveyed accelerometers the following objectives are set in developing this study: (1) To adopt a cascaded architecture of compact accelerometer sections of high stiffness-to-mass ratio to provide the capability to increase the sensor sensitivity to the desired level and maximize its detection speed, (2) To adopt the use of a simple suspended Si beam as a spring member in the suspension system relying principally on the bending elasticity of the beam to increase the bandwidth response of the suspension system to close values of its fundamental resonant frequency, (3) To base the acceleration detection method on modulating the phase of a single tone optical carrier in a Mach-Zehnder Interferometer (MZI) detection processor integrated on the sensor chip providing the capability to eliminate the effects of the carrier intensity fluctuation and temperature variation, (4) To adopt the use of electrostatic actuation of the beam springs used in the suspension system of the sensor to adjust its sensitivity according to the measured acceleration using applied voltages that would also enable programming the vibratory electromechanical characteristics of the sensor, and (5) To adopt the use of a cost-effective SOI integration template with under-etching fabrication step in the box layer to advantage the low optical losses and compact size of the components used in constructing the sensor.

SOI-based MEMS/NEMS devices advantage the underetching fabrication step in the box layer to render part of the Si optical guiding structure free to move under the effects of electrostatic, thermoelastic, and inertial forces. The electrostatic actuation is more power-efficient and faster in operation than thermal actuation. In this study it is determined that the suspended slot waveguide is the best candidate to ultimately play the following roles: (1) Acting as a spring member in the suspension system of the proof mass, (2) Providing electrostatic actuation for the suspension system, and (3) Acting as a phase shift element in the photonic acceleration detection circuit. The simple beam structure of the suspension slot waveguides allows for adapting the stiffness of the suspension system to the desired application by just adjusting the length of the suspension slot waveguides. The small width of $100 \mathrm{~nm}$ at the standstill of the slot between the two beams of the waveguide allows effective electrostatic actuation between the suspended beams at low applied voltages and minimizes the crosstalk effect of the electrostatic forces between the different well-isolated spring members of the integrated chip. Although the suspended slot waveguide allows limited mechanical displacements of the proof mass, the refractive index of the suspended slot waveguide is intensely modulated by modulating the excursions of the beams of the waveguide due to the applied acceleration. This allows achieving the phase shift needed to measure the desired maximum applied acceleration by increasing the length of each suspended slot waveguide and by using more cascaded sections of suspended slot waveguides in the phase shift line of the sensor. Increasing the number of cascaded sections of shorter suspended slot waveguides also helps to maximize the bandwidth response of the sensor. The use of the suspended slot waveguide helps to increase the bandwidth response of the accelerometer and provides dynamic control of its sensitivity.

The utilization of the under-etched suspended slot waveguide as an electrostatically controlled phase shift element was reported in the literature, e.g. by Acoleyen et al. [22, 23] and Chiu et al. [24]. A novel class of the NEMSoperated phase shifter biased through a single electrode located at one side of the structure was proposed by Hussein et al. [25] and was used in studying the construction of MZI phased array processors. This paper investigates the utilization of the suspended Si beams of the NEMS-operated phase shifter as adaptive voltage-controlled spring elements attached to an in-plane suspended Si mass actuator in a phased MZI photonic detection system. The proposed photonic sensor has the advantage of structure simplicity among all other previously reported counterparts, and its design can be tailored to match applications with increased bandwidth demand. The anchoring spring structure is simple and well defined in terms of just the length of the suspended Si beams. Based on the weight of the used suspension proof mass, the length of the straight Si spring element can be virtually optimized to match applications ranging from the detection of harsh impact accelerations in several thousands of $\mathrm{ms}^{-2}$ down to tiny vibrations with peaks in sub- $\mathrm{ms}^{-2}$. The distributed electrostatic force applied between the suspended Si beams of the slot waveguide is used to provide feedback motion control over the mass-spring-damper suspension system. The application of feedback voltage to hold the actuator mass near a standstill is shown useful to increase the acceleration dynamic range. Another important advantage of the distributed electrostatic force is to control the spring constant of the suspended Si beam element. This is shown to significantly increase the accelerometer sensitivity when differentially tensioning the spring elements. As an application example, the sensitivity of an ultrasonography sensor can be adjusted to enable listening to different level ultrasound wave reflections from the boundaries between the tissues of an examined patient. Also interestingly as in piezoelectric devices, the distributed electrostatic force 
between the spring Si beams can be used to generate tiny sub-nanometer vibrations of the seismic mass albeit at low power levels. The MZI-based accelerometer also provides simple detection by converting the phase modulation achieved in the phase shift line of cascaded suspended slot waveguides into either direct intensity modulation of the optical carrier over a wide band of frequencies or by wavelength steering the periodic transmission responses of the $\mathrm{MZI}$-based filter resulted when long delay lines are included in the two arms of the MZI circuit. The acceleration-related wavelength shift of the transmission responses is also converted into intensity modulation of the single tone optical carrier. By comparing the optical carrier which is intensity-modulated due to the applied acceleration to the original signal injected from the optical source the intensity fluctuations of the optical source and those due to vibrations in the optical coupling structures are removed. This provides cost-effective detection of the acceleration at robust performance. The proposed accelerometer uses only three electrodes that are simply aligned in the chip without the need for crossover wirings, and it uses high-efficiency photonic components. The nanometer range motion of the proof mass which results in intense modulation of the effective index of the suspended slot waveguide is expected to make it possible to listen to sub-ms $\mathrm{m}^{-2}$ vibrations and measure tiny picometer displacements when providing a possible external excitation to the anchored mass. The optimization of speed and sensitivity of the proposed accelerometer can be enhanced as outlined in the next section by cascading more sensor sections within the available patterning area of the integration chip (typically around $2 \times 2 \mathrm{~mm}^{2}$ for standard foundries). All components of the proposed accelerometer are integrated in-plane on a standard SOI chip, and the mechanical design of its Si structure is sturdy. Additionally, the differential modulation of the effective refractive index of the phase shift elements in the MZI phase-detection system enhances sensing the acceleration only in the direction of interest and differentially counterbalances vibrations in the orthogonal directions and those due to torsion forces.

Table 1 compares some of the distinguishable aspects of the accelerometer proposed in this study with several other important contributions found in the literature. The most interesting points this study provides are the flexibility in design to match the needs of a wide spectrum of applications and the major improvements in controlling the sensitivity and increasing the bandwidth of the proposed accelerometer. The characteristics of the sensor can be varied in design over a wide range by changing the length of the suspended Si beams and the number of cascaded sections to virtually match any desired application. The pro parameters of the proposed sensor exceed all those reported in Table 1 from previous studies. For example, the optical sensitivity for nine cascaded sections increased by applying equal electrode voltages to measure small accelerations is more than ten times that reported by Ahmadian et al. [21] and it is tunable. The proposed sensor also responds to sinusoidal actuation forces from $\mathrm{OHz}$ to the frequency of resonance of the suspension slot waveguides affected by their length and the applied electrode voltages. Depending on the required application the length of the suspended slot waveguides is recommended in this study within the range of 5.4-25 $\mu \mathrm{m}$. This sets the available bandwidth of the proposed sensor within the range of $25.2-250 \mathrm{kHz}$ at reset voltages which cannot be matched easily by many previously reported MEMS sensors.

The rest of the paper is organized systematically. In Sect. 2, the structure of the proposed accelerometer is detailed, and the principle of operation is explained. In Sect. 3, one section of the sensor is modeled and analyzed. The numerical analyses are then used in Sect. 4 to theoretically demonstrate the performance of the accelerometer handled in general for varying applications. At last, Sect. 5 presents general conclusions and suggestions for future work.

\section{The principle of operation and structure of the accelerometer}

\subsection{The mass-spring-damper model}

The principle of operation of accelerometers relies on the traditional model of a suspended test mass $m$ anchored with a spring of constant $k$ and damper of constant $b$. In SOI-based accelerometers, the spring effect is introduced due to the elasticity of the anchoring Si flexure. The damping effect is introduced due to: (1) The viscosity of the air around the test mass and other parts of the suspension system, (2) The compressibility damping of the air film around the movable parts and between the movable and fixed parts of the structure [29, 30], and (3) The thermoelastic damping of the suspension beams [31]. The viscosity damping dominates at low vibration frequencies where the air in the pockets finds enough time to escape, whereas the squeeze-film air damping becomes more effective at high vibration frequencies where the air is rather squeezed and sucked. The thermoelastic damping may become intense when loading the suspension beams with heavy vibrations.

In this work, the motion direction is taken along the $x$ -axis. When the template carrying the suspension system is subjected to an acceleration of $a \boldsymbol{u}_{a}$, where $\boldsymbol{u}_{a}=-\boldsymbol{u}_{x}$ is a 
Table 1 Comparing some aspects of the proposed accelerometer with several other contributions in the literature

\begin{tabular}{|c|c|}
\hline Reference, Year & Reported Aspects of the Study \\
\hline$[21], 2018$ & $\begin{array}{l}\text { Operation principle: Intensity modulation of the light } \\
\text { Mechanical sensitivity: } 1019 \mathrm{~nm} / \mathrm{g} \\
\text { Optical sensitivity: } 145.7 \% / \mathrm{g} \\
\text { Detection range: } \pm 10 \mathrm{~g} \\
\text { Resonant frequency: } 15.553 \mathrm{kHz} \\
\text { Available bandwidth: } 7 \mathrm{kHz}\end{array}$ \\
\hline$[26], 2016$ & $\begin{array}{l}\text { Operation principle: Intensity modulation of the light } \\
\text { Mechanical sensitivity: } 119.21 \mathrm{~nm} / \mathrm{g} \\
\text { Optical sensitivity: } 0.496 \% / \mathrm{g} \\
\text { Detection range: } \pm 38 \mathrm{~g} \\
\text { Resonant frequency: } 1.444 \mathrm{kHz} \\
\text { Available bandwidth: } 1 \mathrm{kHz}\end{array}$ \\
\hline$[27], 2011$ & $\begin{array}{l}\text { Operation principle: Variable optical attenuator (VOA). Intensity modulation of the light by a movable Bragg mirror in } \\
\text { reference with an un-modulated optical carrier } \\
\text { Integration template: SOI-based } \\
\text { Resonant frequency: } 4.1 \mathrm{kHz} \\
\text { Linear detection range: } \pm 0.7 \mathrm{~g}\end{array}$ \\
\hline$[28], 2012$ & $\begin{array}{l}\text { Operation principle: Modulating the resonance frequency of an in-plane Fabry-Pérot microcavity (FPM) structure with two } \\
\text { distributed Bragg reflectors } \\
\text { Integration template: SOl-based } \\
\text { Mechanical sensitivity: } 90 \mathrm{~nm} / \mathrm{g} \\
\text { Other attributes: Used serpentine springs in the suspension system. Torsion forces may affect the measured acceleration. } \\
\text { Requires costly spectral interrogation methods in detection }\end{array}$ \\
\hline$[4], 2015$ & $\begin{array}{l}\text { Operation principle: Low-power highly sensitive capacitor accelerometer } \\
\text { Limitations: The effect of the high parasitic capacitance of the used bulk micromachined structure is unavoidable. Noise } \\
\text { power optimization and compensation are needed to improve the performance of the device } \\
\text { Rest capacitance: } 20 \mathrm{pF} \\
\text { System sensitivity: } 1.95 \mathrm{~V} / \mathrm{g} \\
\text { Detection range: } \pm 1 \mathrm{~g} \\
\text { Resonant frequency: } 1.5 \mathrm{kHz} \\
\text { Device size: } 2 \times 2.5 \times 0.49 \mathrm{~mm}^{3}\end{array}$ \\
\hline$[13], 2020$ & $\begin{array}{l}\text { Operation principle: Optical MEMS Fabry-Pérot accelerometer } \\
\text { System sensitivity: } 183.793 \mathrm{~V} / \mathrm{g} \\
\text { First resonant frequency: } 89 \mathrm{~Hz} \\
\text { Available bandwidth: } 29.3 \mathrm{~Hz} \\
\text { Other attributes: Used a G-shaped cantilever suspension system. Prone to many mechanical vibration modes. Requires } \\
\text { costly packaging }\end{array}$ \\
\hline This study, 2021 & $\begin{array}{l}\text { Operation principle: Phase modulation of the optical carrier operating at } 1.55 \mu \mathrm{m} \text { wavelength } \\
\text { Detection system: An integrated optical MZI structure is used to convert the phase modulation into intensity modulation } \\
\text { of the light. Simple detection is needed utilizing photodetectors and intensity noise cancellation of the optical carrier } \\
\text { Integration template: SOl-based utilizing under Si etching in the box layer } \\
\text { Structure: All in-plane components on a cost-effective integration template } \\
\text { Architecture: Cascading of compact wide bandwidth sensor sections } \\
\text { Dynamic control: Two voltages are used to control the sensitivity and increase the acceleration measurement range of the } \\
\text { sensor utilizing electrostatic forces } \\
\text { Maximum measurable acceleration recommended in design at reset voltages: } \pm 10.2 \mathrm{~g} \text { to } \pm 1020.4 \mathrm{~g} \\
\text { Mechanical sensitivity: } 0.0588-65.75 \mathrm{~nm} / \mathrm{g} \text { for nine cascaded sections (voltage controlled) } \\
\text { Optical sensitivity: } 0.239-1489.8 \% / \mathrm{g} \text { for nine cascaded sections (voltage controlled) } \\
\text { Detection range: } \pm 0.0671 \mathrm{~g} \text { to } \pm 418.1 \mathrm{~g} \text { (voltage controlled) } \\
\text { Available bandwidth: Up to the fundamental resonant frequency within the range } 25.2-250 \mathrm{kHz} \text { at reset voltages depend- } \\
\text { ing on the length of the suspension slot waveguides } \\
\text { Integration chip patterning area: } 2 \times 2 \mathrm{~mm}^{2} \\
\text { Other attributes: Focused to measure acceleration along one axis }\end{array}$ \\
\hline
\end{tabular}

unit vector in the negative direction of the $x$-axis, then the test mass makes a deflection $x$ along the $x$-axis. The deflection in the test mass is referenced to the template that carries the suspension system. The full-scale deflection $\pm x_{m}$ that can be made by the test mass depends on the suspension system structure. The relative deflection in the test mass $\chi=x / x_{m}$ has a normalized range of \pm 1 . The $2^{\text {nd }}$ order motion equation of the suspension system is given by: 
$m \chi^{\prime \prime}+b \chi^{\prime}+k \chi=\max _{m}^{-1}$

where the apostrophe and double-apostrophe indicate the first-order and second-order time-derivatives, respectively. The Laplace transform of Eq. (1) yields the transfer function of the suspension system in the s-domain:

$\frac{\chi}{a}=\frac{x_{m}^{-1}}{s^{2}+2 \zeta \omega_{n} s+\omega_{n}^{2}}$

$\omega_{n}=\sqrt{k m^{-1}}, \zeta=0.5 b / \sqrt{k m}$

$\omega_{n}$ is the fundamental angular frequency and $\zeta$ is the damping factor. The frequency response of the suspension system to a time-varying acceleration depends on the location of the system transfer function poles of Eq. (2) in the s-plane: $p_{1,2}=\zeta \omega_{n}\left[-1 \pm\left(1-\zeta^{-2}\right)^{0.5}\right]$. A suspension system is under-damped, critically-damped, and overdamped when $\zeta<1\left(b<2(\mathrm{~km})^{0.5}\right), \zeta=1\left(b=2(\mathrm{~km})^{0.5}\right)$, and $\zeta>1\left(b>2(\mathrm{~km})^{0.5}\right)$, respectively, and the transfer function of Eq. (2) would have two complex-conjugate poles, similar real poles, and different real poles, accordingly. Theoretically, if no damping exists $(b=0, \zeta=0)$ then the suspension system becomes unstable and can oscillate forever when triggered by an impulse acceleration. The amount of displacement the suspended seismic mass makes in response to an applied acceleration determines the mechanical sensitivity of the sensor. Based on Eq. (2) at $\omega=0$ the mechanical sensitivity may be taken as $\Delta \chi / \Delta a=x_{m}^{-1} / \omega_{n}^{2}=x_{m}^{-1} m / k$. This shows that the mechanical sensitivity increases with the increase of the seismic mass and decreases with the increase of the suspension structure rigidity. In general, the characteristics of $\chi, f_{n}$, and $\Delta \chi / \Delta a$ may be affected by the actuation forces applied to the suspension system. The fundamental resonant frequency $f_{n}$ determines the speed at which the suspension system can track acceleration changes with time. A higher value of $f_{n}$ results in a higher bandwidth response of the sensor. Increasing the seismic mass results in increasing the sensitivity, but it also decreases the bandwidth response of the suspension system and vice-versa. The choice of $m$ in a single-unit suspension accelerometer, therefore, is an optimization between speed and sensitivity. Once the value of $m$ is chosen for a certain application such that the maximum deflection in the suspension system covers the expected maximum acceleration, then adaptive control of the spring constant $k$ may be deployed to accurately detect smaller accelerations. A smaller value of $k$ adjusted when electrostatically tensioning a system of two differential springs results in increasing the sensitivity of the sensor at the expense of decreasing its bandwidth response. The goal of achieving both high sensitivity and wide bandwidth response may be reached by cascading several wide-bandwidth low-sensitivity accelerometer sections. This target is practically accountable for utilizing compact sensor elements of simple structure.

\subsection{The principle of operation and detection system}

The principle of operation relies on converting the inertial force due to accelerating the seismic mass into phase modulation of the light passing into a suspended slot waveguide. An SOl-based slot waveguide consists of two Si beams of high index separated by a gap of low index material usually taken as air. The fundamental optical mode in a slot waveguide is enhanced and guided in the low index region as an evanescent field [32]. The refractive index of the slot waveguide is dependent on the width of the slot between the beams. The modulation of the slot width resulting from laterally deforming the suspended beams of a slot waveguide modulates the refractive index and results in introducing a phase shift difference into the propagating light $[22,23]$. The deformation of the suspended beams may be affected by the following forces: (1) The Casimir-Lifshitz force [33, 34], (2) The optomechanical force [35, 36], (3) The electrostatic force [22-24], (4) The inertial force, and (5) The thermoelastic force. In the absence of strong optomechanical, electrostatic, and inertial actuation forces the Casimir-Lifshitz force plays an important role during the fabrication process and may cause bonding of the movable parts when small distances separate them. The inclusion of motion restrictors in the design of the sensor structure may alleviate this issue. Several researchers have studied the harnessing of the attractive and repulsive distributed gradient optomechanical force due to the interactions of the evanescent electromagnetic fields [37-39]. The effective optomechanical actuation of SOI-based slot waveguides requires large optical power in the $\mathrm{mW}$ range. In a recent study, Feng et al. [40] compared the effects of the Casimir force, optomechanical force, and electrostatic force considering a slot waveguide with dimensions comparable to the slot waveguide used in this study. The simulation results of Feng et al. confirm that the Casimir force is a good magnitude less than the electrostatic force when the gap width of the slot waveguide is kept large enough (taken above $20 \mathrm{~nm}$ in this study) and that the optomechanical force is negligible compared to the electrostatic force when the optical power is kept well low in the $\mu \mathrm{W}$ range (below $100 \mu \mathrm{W}$ ). Keeping the optical power low in the suspended slot waveguides also minimizes the incidental thermoelastic stress due to the optical absorption loss. The actuation of the slot waveguides used in the sensor proposed in this study relies principally on the inertial and electrostatic 
forces and ignores all other possible forces in the analysis assuming the use of a low $\mu \mathrm{W}$ range optical power. The distributed electrostatic force results due to the application of a voltage between the suspended Si beams of the slot waveguide. The inertial forces include: (1) The force applied by the accelerated seismic mass attached to one of the two beams of the slot waveguide, (2) The acceleration force of the distributed mass of the Si beam itself and other parts of the suspension system, and (3) The gravitational acceleration force. The inertial forces contributed by the seismic mass and by the distributed mass of the beams and other movable parts of the suspension system contribute to the measurement of the acceleration in the direction of interest. The effect of the gravitational force depends on the actual orientation of the device about the ground. The gravitational force may add a DC component to the measured acceleration which may be either subtracted in the detection algorithm or be compensated by electrostatic forces applied between the beams of the slot waveguides used in the suspension system. When the proposed sensor template is kept horizontal to the ground the gravitational force does not affect the measured acceleration but the seismic mass weight pulls the suspension system down. For these reasons to reduce the gravitational force effect the length of the suspended slot waveguides should be kept small enough such that the maximum measurable acceleration at full-scale deflection of the seismic mass at reset voltages has to be many magnitudes greater than the gravitational acceleration. This study recommends setting the maximum measurable acceleration at reset voltages of the electrodes within the range of $10^{2}-10^{4} \mathrm{~ms}^{-2}$. This also assures that the strength of the suspension system is capable of carrying the seismic mass weight during and after the fabrication process.

The suspended slot waveguide adopted in this study is illustrated in Fig. 1. This component plays the following roles: (1) Acting as a phase shifter to the light propagating through it, (2) Acting as a member of the suspension system to hang the seismic mass, and (3) Acting as an electrostatic-controlled electromechanical spring. Each beam of the slot waveguide has a cross-section area of $W_{g s} \times h$, where $h$ is the full thickness of the Si core layer and $W_{g s}$ is the width of the beam. The two suspended beams are separated by a slot of width $W_{s}$ which might be modulated by the inertial and electrostatic forces. The total length of the suspended slot waveguide is $L_{s}$. The lower beam shown in the figure is attached at its middle point to the seismic mass by a stem of narrow width used to both hang the seismic mass and to transfer the acceleration force from the seismic mass to the beam. The lower beam is also connected to the ground electrode of the sensor. The upper beam in the figure is attached at its middle point by a non-movable narrow width stem used to advantage both connecting the beam to an electrode and split the total length of the beam into two suspended halves. This reduces overwhelming the excursion of the upper beam under the influence of the electrostatic force. When acceleration is applied the inertial force of the seismic mass in principal mechanically modulates the excursion of the lower beam. The inertial force of the distributed mass of both beams also contributes to modulating their excursions but at a lower degree compared to the effect of the heavy seismic mass. As a result, the slot width between the beams is modulated in proportion to the magnitude and direction of the applied acceleration. On the other hand, when voltage is applied to the upper beam the distributed attraction electrostatic force between the two beams contributes to reducing the modulated gap width. The electrostatic force in this work is utilized to either provide negative feedback control for the sensor system holding the seismic mass at a position close to a standstill or to increase the sensor sensitivity.

To illustrate the overall operation the schematic diagram in Fig. 2 shows the parts of the sensor system. The light may be taken from a laser source built on the integration template utilizing CMOS compatibility [41-43]. The light may also be taken from an external tunable laser source with polarization control equipment and fiber
Fig. 1 The suspended slot waveguide as a photonic phase shifter and electromechanical suspension spring

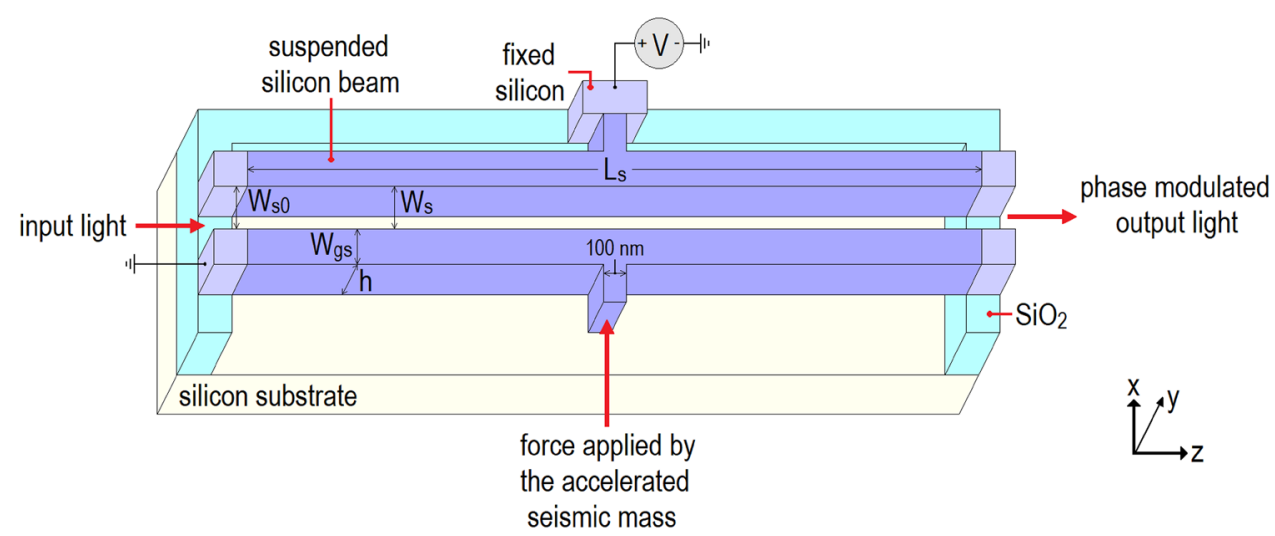


optic coupling to the sensor chip following lab protocols to keep the fabrication cost of the sensor low. In such a case either edge couplers or surface grating couplers are required on the integration template to couple the light from the input fiber to the sensor chip [44-46]. The input fiber has to be relatively attached to the sensor chip to reduce coupling vibrations such as by inserting the fiber tip into a groove made at the edge of the integration template or by using a ridge fiber coupling attachment of high stiffness. The use of on-chip and off-chip active coupling alignment to the input fiber may also alleviate this issue for lab testing. For industrial production integrating the lasing source with the sensor has the advantages of cutting on the cost and eliminating the intensity fluctuation of the input light due to vibrations in the coupling fiber. The integration of a programmable polarization controller $[47,48]$ on the sensor chip to couple the desired fundamental mode is also advantageous to avoid the cost of expensive external polarization controllers. The different photonic components on the integration template of the sensor are connected by waveguides. After the fundamental optical mode is coupled it is equally divided by a splitter into a reference and sensing optical carriers. The light splitter is implemented here as a $2 \times 2 \mathrm{MMI}$ multimode interference (MMI) coupler. MMI couplers are ubiquitous components that rely on the self-imaging principle of the input mode $[49,50]$ and their imbalances and excess losses may be improved by using tapers at their input and output ports [51]. The reference optical carrier is converted into a reference voltage $V_{r}$ by a photonic detector (PD). All the PDs used in the system may also be integrated into the sensor template for an accurate detection process. The sensing optical carrier is applied to the MZI core network of the sensor which is composed as shown in the symbolic diagram of Fig. 2 from a splitter and combiner $2 \times 2$ MMI couplers interconnected by a network of waveguides, phase shifters, and delay lines. The seismic mass shown in this figure has two continued sections each of mass $m$. At least two cascaded sections must be included for sturdy suspension of the seismic mass. The four suspension slot waveguides used to hang the seismic mass are placed in the two arms of the MZI processor. When the seismic mass is accelerated along the positive $x$-direction the phase shifters in the upper arm produce a total phase difference $\Delta \phi_{1 t}$ larger than the total phase difference $\Delta \phi_{2 t}$ produced by the phase shifters in the lower arm. This is due to decreasing the modulated slot width of the phase shift components in the upper arm and increasing the modulated slot width of the phase shift components in the lower arm. The transmission response of the MZI processor represents the ratio of the light power at one of its outputs to the light power at one of its inputs. The transmission responses of the MZI processor may be set broad over a wide range of wavelengths in the $C$-band when the difference in length between the delay lines $\Delta L=L_{1}-L_{2}$ is taken short in the subwavelength range to introduce the phase shift needed to calibrate the power of outputs $\mathrm{O}_{1}$ and $\mathrm{O}_{2}$ of the MZI core processor to be equal at zero

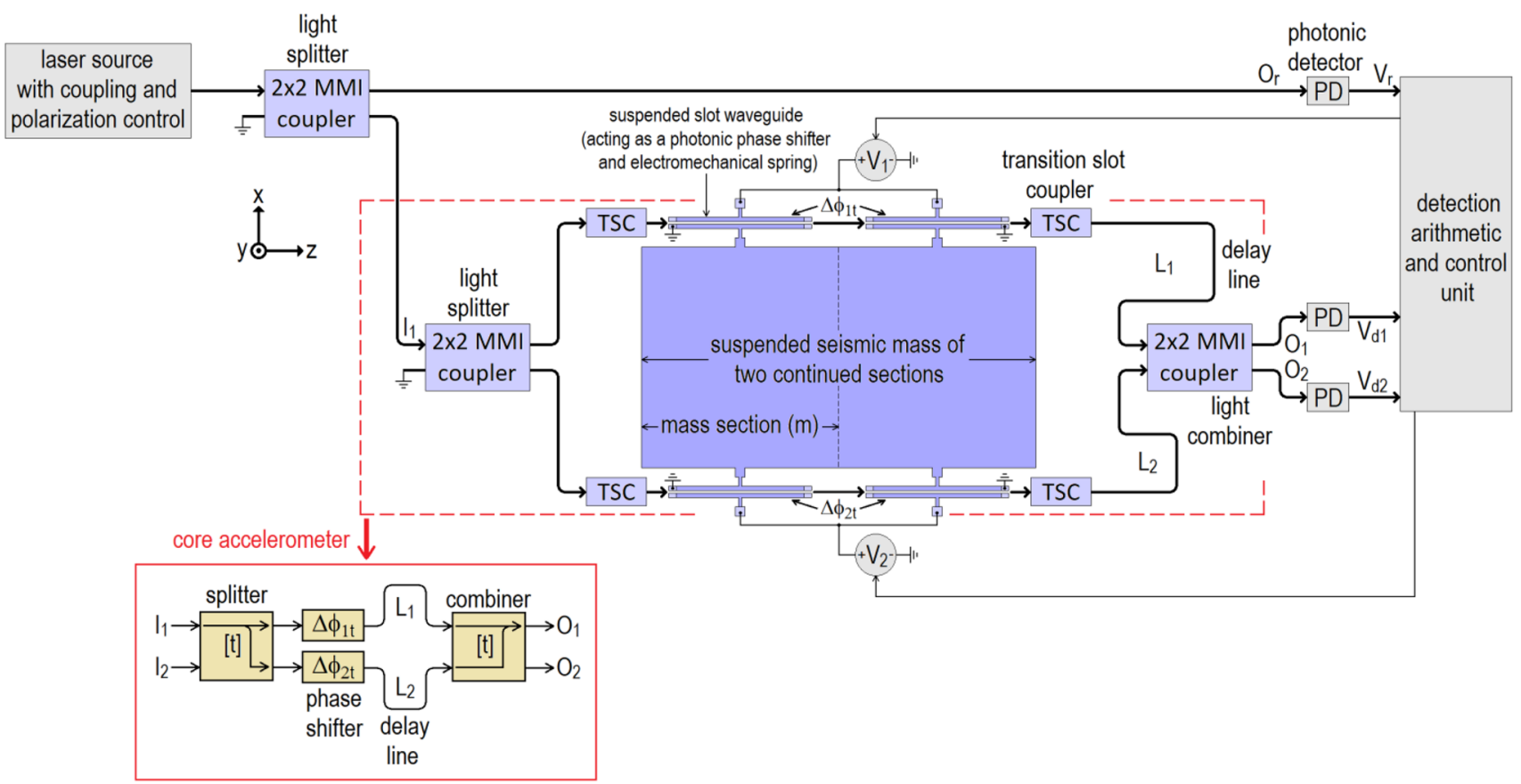

Fig. 2 Schematic diagram illustrating the parts of the sensor system 
acceleration. This calibration in design is necessary so that the sensor can measure the acceleration in both the positive and negative directions of the $x$-axis. The accuracy of this calibration requires a subwavelength precision in the fabrication process. The length imbalance $\Delta L$ may also be taken long such that the transmission responses of outputs $O_{1}$ and $O_{2}$ become periodic functions of wavelength in the $\mathrm{C}$-band. The acceleration-induced total phase difference $\Delta \phi_{t}=\Delta \phi_{1 t}-\Delta \phi_{2 t}$ either modulates the transmissions of outputs $O_{1}$ and $O_{2}$ when the MZI is taken as a broadband processor or it results in wavelength shifting the periodic transmission responses of the outputs when the MZl is taken for a long value of $\Delta L$. To connect the slot waveguides to the non-slot waveguides two transition slot couplers (TSCs) are used in each arm of the MZI processor. The voltages $V_{1}$ and $V_{2}$ applied to the exterior beams of the suspended slot waveguides are used to control the electromechanical characteristics of the sensor as explained in this study. The optical outputs $O_{1}$ and $O_{2}$ are converted into the voltages $V_{d 1}$ and $V_{d 2}$, respectively, that are used by the arithmetic unit to detect the acceleration. The detection voltage corresponding to the difference between $V_{d 1}$ and $V_{d 2}$ is normalized to $V_{r}$ to remove the intensity fluctuation of the light pumped from the optical source such that the detected acceleration only depends on the phase difference modulated by the phase shift elements.

Typical simulation of the transmission response of the $\mathrm{MZI}$ core processor in the broadband regime is depicted in Fig. 3 taken for input $I_{1}$ and outputs $O_{1}$ and $O_{2}$. The path length imbalance $\Delta L$ in this simulation is taken in the subwavelength dimension as $180 \mathrm{~nm}$ to calibrate the sensor at zero acceleration $\left(\Delta \phi_{t}=0^{\circ}\right)$ such that the transmissions of outputs $O_{1}$ and $O_{2}$ are equal. When the seismic mass is accelerated into the positive $x$-direction $\Delta \phi_{t}$ becomes positive and the transmission of output $O_{1}$ becomes larger than that of output $\mathrm{O}_{2}$ and therefore $V_{d 1}>V_{d 2}$ detects the acceleration in the correct direction. Similarly, when the seismic mass is accelerated into the negative $x$-direction it becomes that $\Delta \phi_{t}<0^{\circ}$, the transmission of $O_{1}$ is smaller than that of $O_{2}$, and $V_{d 1}<V_{d 2}$ which also detects the acceleration in the correct direction.

In the periodic regime, Fig. 4 depicts a typical simulation of the transmission responses of the MZI core processor taken for a long path length imbalance of $\Delta L=197.91 \mu \mathrm{m}$. The upper part of this figure is simulated for $\Delta \phi_{t}=0^{\circ}$ when no acceleration is applied. The input narrow band optical carrier is set to operate at $1550 \mathrm{~nm}$ such that it matches the wavelength point at which the transmissions of outputs $\mathrm{O}_{1}$ and $\mathrm{O}_{2}$ are equal at zero acceleration. When the maximum measurable acceleration is applied to the seismic mass into the positive $x$-direction for which case $\Delta \phi_{t}=90^{\circ}$ both the transmission responses of outputs $\mathrm{O}_{1}$ and $\mathrm{O}_{2}$ are steered to the right side by quarter

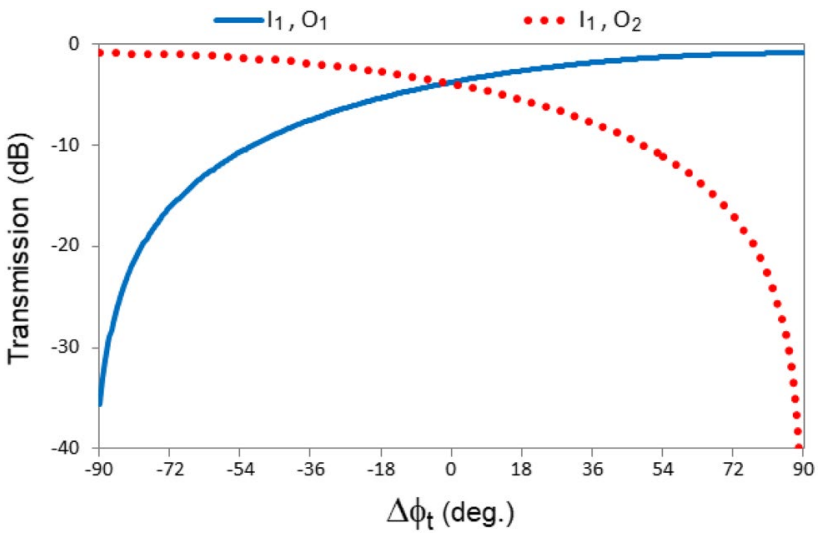

Fig. 3 Transmission response of the MZI core processor for input $I_{1}$ and outputs $O_{1}$ and $O_{2}$ versus the total phase shift difference $\Delta \phi_{t}$. $\Delta L=180 \mathrm{~nm}$

the free-spectral-range (FSR) wavelength as in the lower part of Fig. 4. In this case the transmission of output $O_{1}$ reaches its maximum value and the transmission of output $\mathrm{O}_{2}$ reaches its minimum value at the wavelength of the optical carrier. When the seismic mass is accelerated into the negative $x$-direction $\left(\Delta \phi_{t}<0^{\circ}\right)$ the transmission responses are wavelength steered to the left side. The wavelength steering of the transmission responses modulates $V_{d 1}$ and $V_{d 2}$ used in the detection of the acceleration. It is also possible to use optical spectral interrogation methods to detect the acceleration based on the wavelength shift of the transmission responses at outputs $O_{1}$ and $O_{2}$ but this requires using more expensive equipment such as a broadband optical source and spectrum analyzer.

To simply illustrate the benefit of applying voltages to the exterior beams of the suspended slot waveguides on increasing the sensor sensitivity parts (a) and (b) of Fig. 5 show representation of the motion behavior when no voltage is applied $\left(V_{1}=V_{2}=0\right)$ and when an equal voltage is applied $\left(V_{1}=V_{2}=V_{c}\right)$, respectively, both taken for the same acceleration of the seismic mass in the positive $x$ -direction. In part (a) the exterior beams stay stationary as no voltage is applied and the interior beams bend in the motion direction of the seismic mass due to elasticity. When the equal voltage $V_{c}$ and no acceleration applied the interior and exterior beams of each of the upper and lower suspended slot waveguides are attracted to each other by the same amount due to the equal distributed electrostatic forces so the seismic mass stays at the standstill position. However, in this case when the seismic mass is accelerated into the positive $x$-direction the modulated slot width between beams $1 a$ and $1 b$ in the figure becomes smaller than that between beams $2 a$ and $2 b$ and therefore the distributed electrostatic force in the upped waveguide increases and the distributed electrostatic force in the 
Fig. 4 Wavelength transmission response of the $\mathrm{MZI}$ core processor. $\Delta \phi_{t}=0^{\circ}$ in the upper part, $\Delta \phi_{t}=90^{\circ}$ in the lower part, and $\Delta L=197.91 \mu \mathrm{m}$
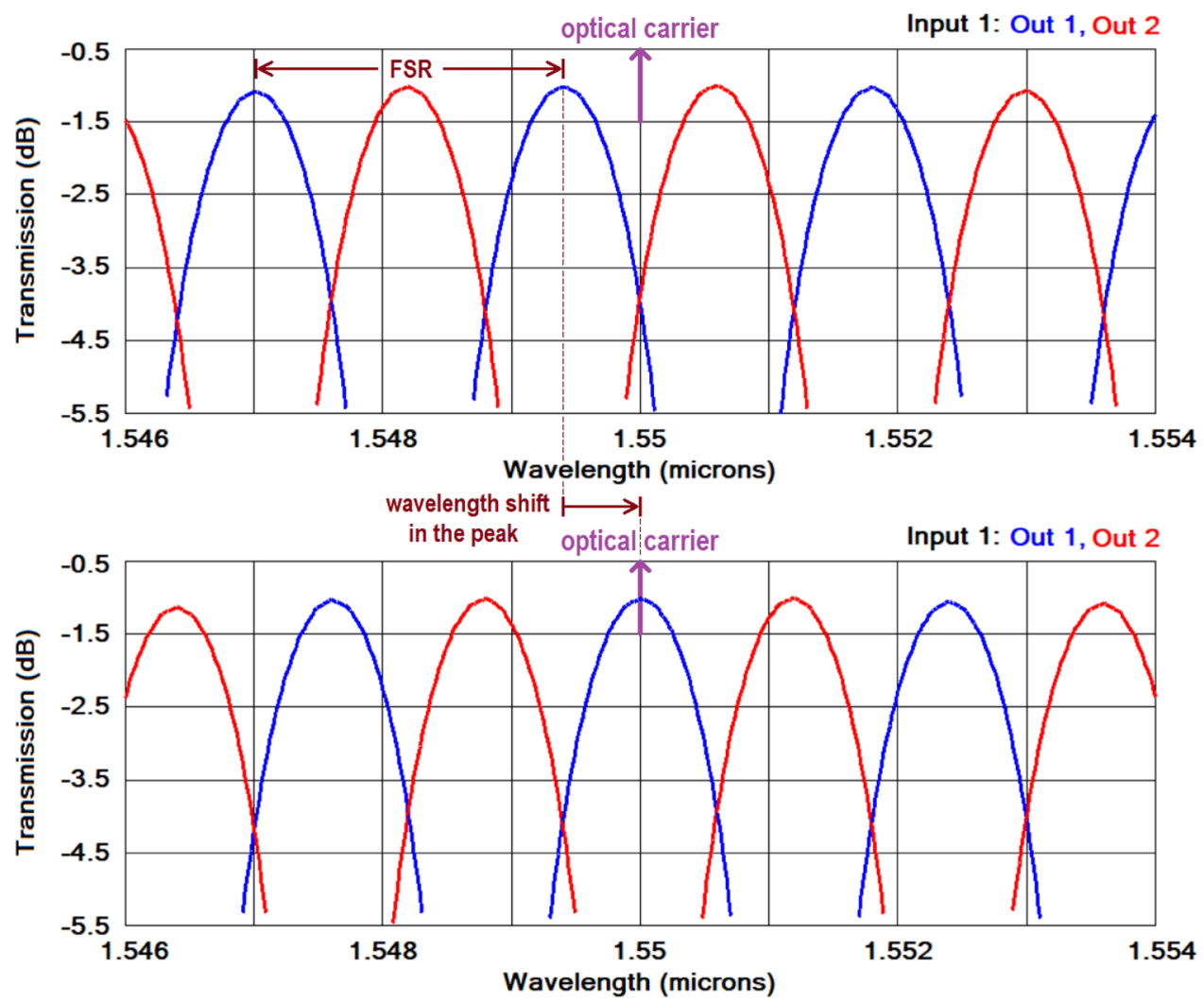

lower waveguide decreases. This effect causes pulling the seismic mass further in the $x$-direction compared to part (a) of the figure and therefore the mechanical sensitivity of the sensor increases by applying an equal voltage. The deformation in the beams of the suspended slot waveguides due to the applied non-zero equal voltage also has the effect of increasing their equivalent length and decreasing their equivalent spring constant. This will be shown in the results of Sect. 4 to have the effect of reducing the fundamental frequency of resonance of the suspension beams. The application of a torsion force results in similar phase-shift differences in both arms of the MZI core processor as explained in part (c) of Fig. 5. This same behavior is true for ignoring accelerations in the $\pm y$ and $\pm z$ directions. This credits the proposed sensor to be focused to measure the acceleration component only in the $\pm x$ direction.

\subsection{The structure and operation of the MZI core processor}

The structure of the core of the proposed nanomachined accelerometer is explained in Fig. 6 . The accelerometer is based on an SOI template with Si base layer thickness of $h=300 \mathrm{~nm}$ albeit other industry standards may be used. The $\mathrm{SiO}_{2}$ box layer area released underneath the $\mathrm{Si}$ base layer is shown in pink color in the figure. The suspended
Si mass actuator block in this figure is composed of two continued sections each of mass $m$. Each mass section is attached at its middle points along the $z$-axis by two $\mathrm{Si}$ stems anchoring it to the midpoints of the Si beams of two suspended slot waveguides. The two NEMS-operated phase shifters of cascaded suspended Si beams and the splitter and combiner $2 \times 2 \mathrm{MMl}$ couplers form a photonic MZI phased acceleration detection circuit. Knowing that the mass density of polysilicon is around $m_{d}=2329 \mathrm{~kg} \cdot \mathrm{m}^{-3}$, a solid square mass section of $m=50 \mathrm{ng}$ requires an area of around $267.5 \times 267.5 \mu \mathrm{m}^{2}$, albeit the possible inclusion of a matrix of vents to ease the under Si etching fabrication step in the box layer would increase the expected total chip area used by the mass actuator. The locations of the splitter and combiner MMI couplers along the lateral $x$-axis are adjustable in design as indicated in the figure to easily create the path length imbalance $\Delta L$ without the need to use extra waveguide bends in the interconnection network. The cascaded suspended slot waveguides of each phase shift line used for one actuator block of continuous mass sections are connected to the rest of the photonic integrated circuit by two transition slot waveguide couplers each of length $L_{t s}=100 \mu \mathrm{m}$ which provides high power coupling efficiency (>95\%) [25].

The dimensions of the transition slot coupler and suspended slot waveguide electromechanical anchoring springs are shown in more detail in Fig. 7. The seismic 


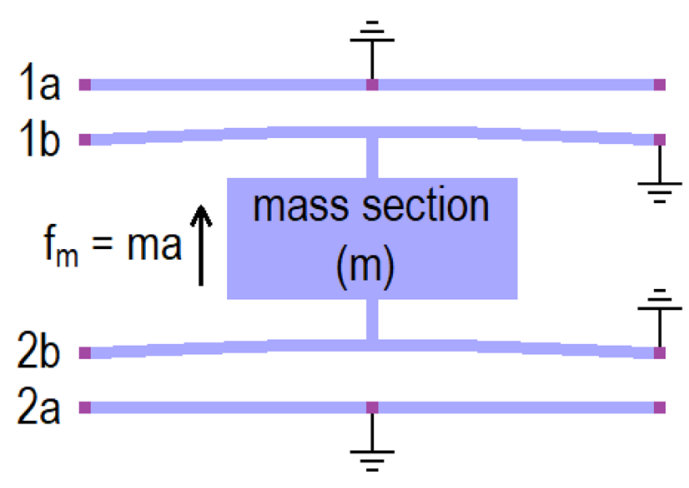

(a)
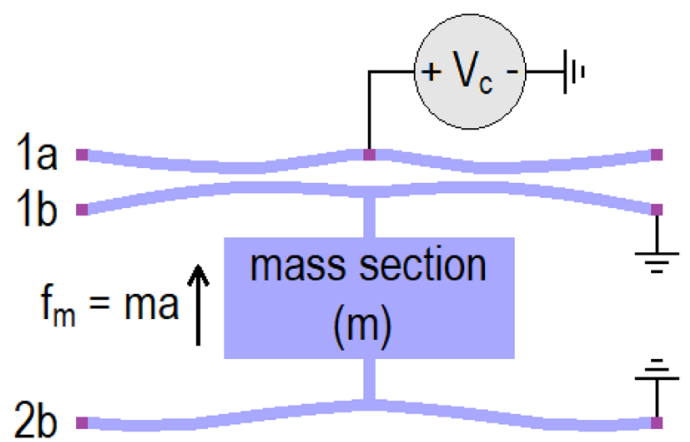

$2 \mathrm{a}$

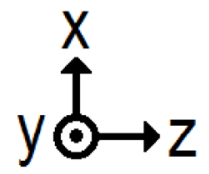

(b)

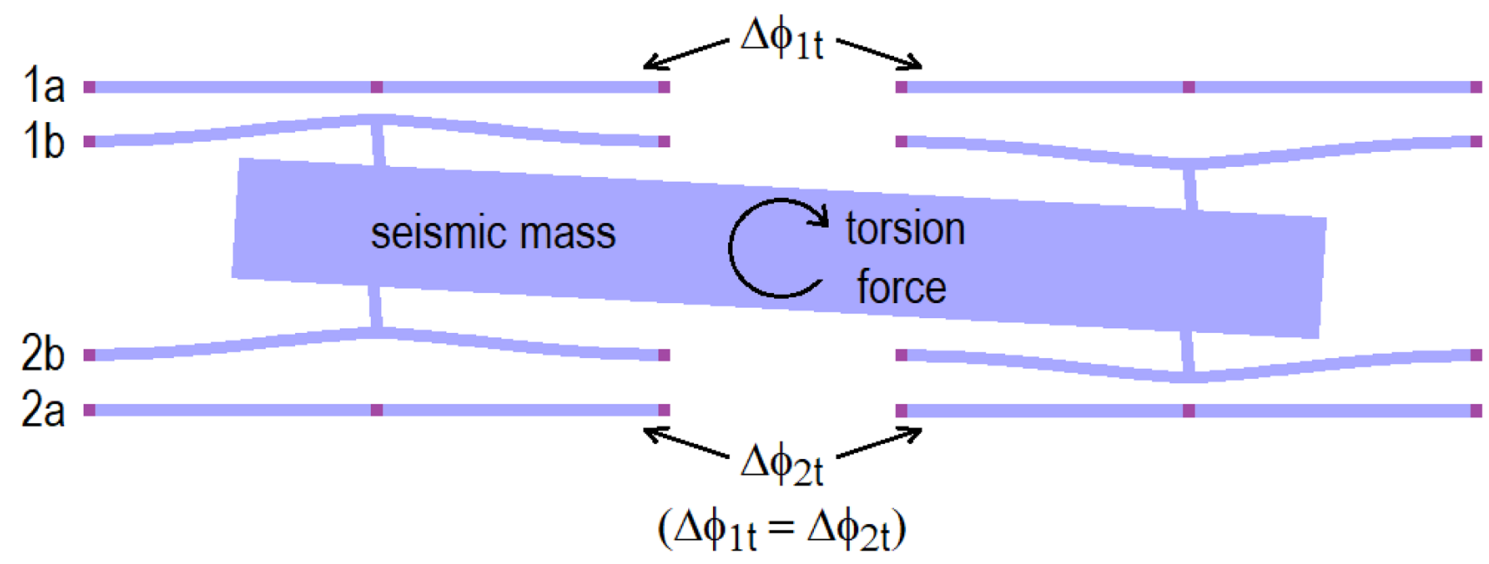

(c)

Fig. 5 Motion responses of the sensor. a: When $V_{1}=V_{2}=0$ and the acceleration force is in the positive $x$-direction, $\mathbf{b}$ : When $V_{1}=V_{2}=V_{c}$ and the acceleration force is in the positive $x$-direction, and $\mathbf{c}$ : When applying a torsion acceleration force

mass is anchored at the mid of the suspended Si beam that is electrically connected through the rest of the $\mathrm{Si}$ structure to the ground electrode. The short straight stem that anchors the seismic mass to the suspended Si beam is just $100 \mathrm{~nm}$ in width to reduce the crosswire loss. The other suspended Si beam of the phase shift element is anchored at its middle point by a fixed stem of $100 \mathrm{~nm}$ width connected to the electrode that is located at one side of the phase shift line. This arrangement not only eases the electrical biasing of the suspended Si beam but also improves the adaptive motion control range by preventing overwhelming the maximum excursion in the $\mathrm{Si}$ beam when applying large electrode voltages. Each $\mathrm{Si}$ beam of the slot waveguide has a width of $W_{g s}=230 \mathrm{~nm}$. The rest-width of the gap between the two suspended $\mathrm{Si}$ beams of the slot waveguide is $W_{s 0}=100 \mathrm{~nm}$. The width of the single-mode waveguide used to interconnect the different photonic circuit components is $W_{g}=350 \mathrm{~nm}$. The design of the $-3 \mathrm{~dB} 2 \times 2 \mathrm{MMI}$ couplers is handy as in [25] providing low figures of imbalance $(<0.1 \mathrm{~dB})$ and excess loss $(<0.2 \mathrm{~dB})$. The application of a voltage between the suspended $\mathrm{Si}$ beams of the phase shift element applies distributed electrostatic force between the inner capacitive plates of the beams. This distributed electrostatic force and the point force applied by the accelerated seismic mass modulate the width $W_{s}$ of the gap between the suspended Si beams of the phase shift element. The modulated excursion width $W_{e x}$ of each suspended $\mathrm{Si}$ beam is measured about the $z$-axis. The modulation of $W_{s}$ 


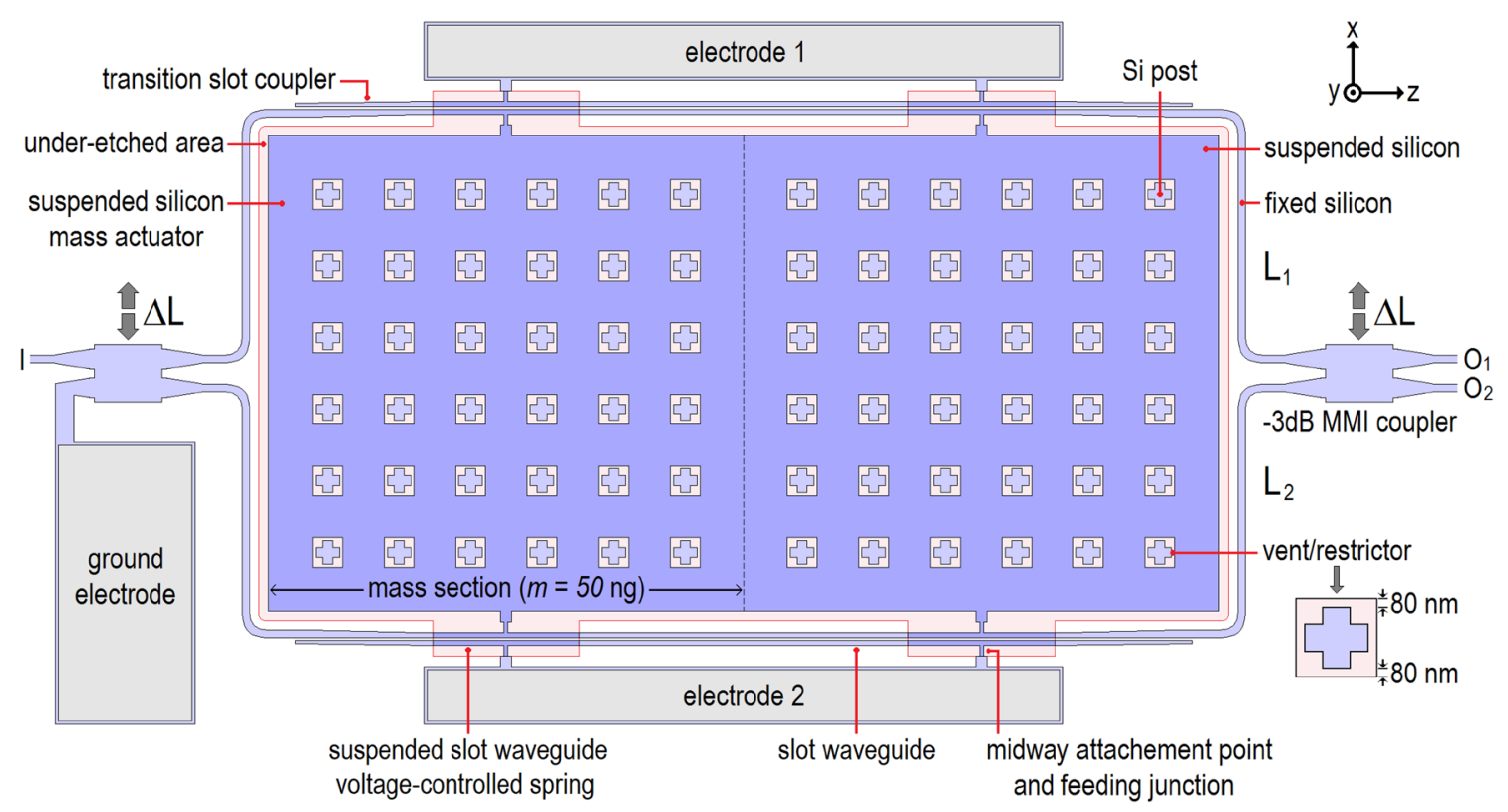

Fig. 6 Schematic diagram of the accelerometer

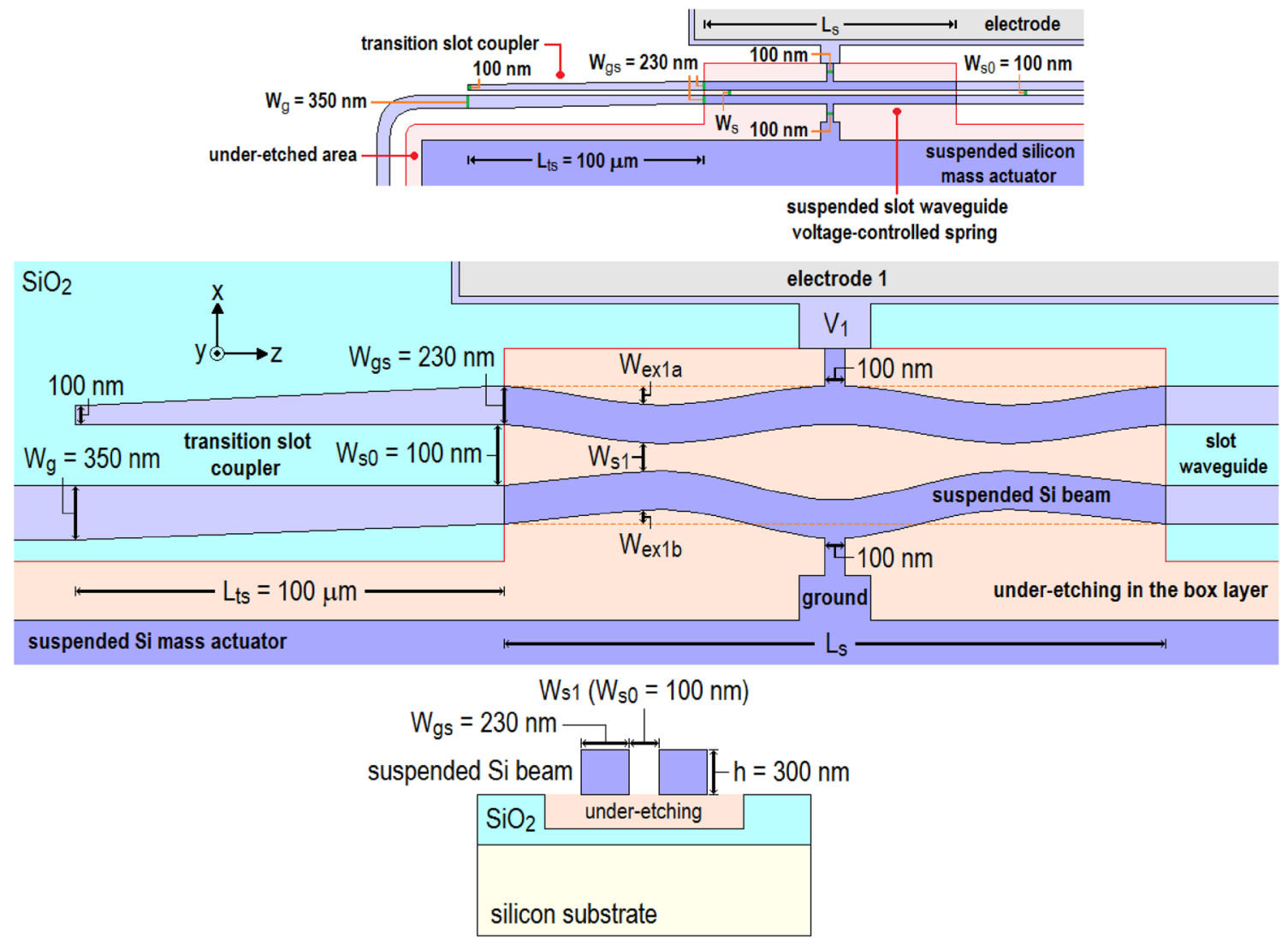

Fig. 7 Dimensions of the transition slot coupler and slot waveguide suspension phase-shift element. The vertical section of the integration template is shown 
introduces an intensified change in the effective refractive index of the slot waveguide.

The two suspended Si beams of the slot waveguide should not come into direct contact with each other to prevent a collapse of the structure when a high potential difference is applied. Consequently, the gap width $W_{s}$ is chosen in design to be within the range of $20-180 \mathrm{~nm}$ maintaining a clearance of $20 \mathrm{~nm}$ between the suspended Si beams. As seen in Fig. 6, Si posts stemming from the box layer can be used as restrictors to force limits on the motion of the test mass along the $x$-axis within the range of $\pm 80 \mathrm{~nm}$. The spring constant $k$ of the suspension system at reset voltages can be simply set in design by adjusting the length $L_{s}$ of the suspended $\mathrm{Si}$ beams of the slot waveguide. A shorter value of $L_{s}$ increases $k$, and hence $f_{n}$ is increased for faster operation, but the sensitivity is decreased. The use of more cascaded accelerometer sections can compensate for the reduction in sensitivity of each wide-bandwidth sensor section for application in ultrasonography. In this respect, more than one mass actuator block each is constructed of multiple continuous mass sections can be utilized as explained in Fig. 8. The multiple seismic mass accelerometer system of this figure uses only three electrodes to bias all of the voltage-controlled suspended Si waveguides no matter how many mass blocks are involved saving on the patterning footprint. The photonic system of the proposed accelerometer uses power-efficient components, and the electromechanical system consumes electric power only when time-varying electrode voltages are applied. Theoretically, no electric power is used while statically tensioning the spring constants of the opposite slot waveguide suspension sections to increase the sensitivity of the sensor. The ground electrode is connected directly to the unused input port of the splitter MMI coupler to keep the accelerometer structure simple.

The simplified motion detection of the accelerometer is based on applying the optical signals at outputs $O_{1}$ and $\mathrm{O}_{2}$ to the PDs attached to those outputs. For acceleration applied into the $x$-direction, the optical response of the $\mathrm{MZI}$ core processor is described using the transfer function method by:

$$
\begin{aligned}
& {\left[\begin{array}{l}
O_{1} \\
O_{2}
\end{array}\right]=\left[\begin{array}{l}
\tau_{11} \\
\tau_{21}
\end{array}\right] \cdot I} \\
& \tau_{11}=t_{11}^{2} e^{j \Delta \psi_{1}}+t_{21}^{2} e^{j \Delta \psi_{2}}, \tau_{21}=t_{11} t_{21}\left(e^{j \Delta \psi_{1}}+e^{j \Delta \psi_{2}}\right)
\end{aligned}
$$

$\Delta \phi_{1}$ and $\Delta \phi_{2}$ are the phase shifts introduced by the differential NEMS-operated phase shift elements anchoring the suspended actuator section of mass $m$. Both $\Delta \phi_{1}$ and $\Delta \phi_{2}$ are functions of the acceleration of the carrying template and the applied voltages $\mathrm{V}_{1}$ and $\mathrm{V}_{2}$. Any accumulated phase shift factor is dropped from the expression of Eq. (3). The total number of cascaded mass sections each of mass $m$ is taken here as $N$. $\lambda$ is the operating wavelength of the optical signal. $n_{e}$ is the effective index of the waveguide from which the path length difference $\Delta L$ of the delay lines is formed. $\tau_{k l}$ is the scattering parameter of the MZI photonic detection circuit taken here for output $O_{k}$ and input $I=I_{1}$. The second input waveguide $I_{2}$ is used to connect the ground electrode to the photonic circuit. $t_{k l}$ is the scattering parameter of the MMI coupler for output $k$ and input $l$. Due to the symmetrical structure of the MMI coupler used in this work, it follows that $t_{k l}=t_{l k}$. The scattering matrix of an ideal MMI coupler with null excess losses and null imbalances is given by:

$\left[t_{k l}\right]=\left[\begin{array}{ll}t_{11} & t_{21} \\ t_{21} & t_{11}\end{array}\right]=\kappa\left[\begin{array}{ll}j & 1 \\ 1 & j\end{array}\right]$

where the common phasor $\kappa$ of an ideal MMI coupler has a magnitude of $|\kappa|=1 / \sqrt{2}$ and phase that is a linear function of wavelength with a negative slope for the increase in wavelength over the C-band. Using Eq. (4) into Eq. (3) yields the simplified scattering relations of the MZI photonic circuit:

$$
\begin{gathered}
\tau_{11}=-\sin (\Delta \psi / 2), \tau_{21}=\cos (\Delta \psi / 2) \\
\Delta \psi=N \Delta \phi+\Delta \varphi, \Delta \phi=\Delta \phi_{1}-\Delta \phi_{2}, \Delta \varphi \\
=\Delta \varphi_{1}-\Delta \varphi_{2}=2 \pi n_{e} \Delta L / \lambda
\end{gathered}
$$

The modulated common phase factor has been removed from Eq. (5) because it has no importance in the detection process. Taking the difference between the voltages $V_{d 1}$ and $V_{d 2}$ produced by the PDs at outputs $O_{1}$ and $O_{2}$, respectively, and normalizing the result to the reference voltage $V_{r}$ produced by the PD at the reference optical carrier $O_{r}$, the normalized detection voltage $V_{d}$ within the range of \pm 1 which detects the phase difference modulated due to the applied acceleration may correspond to the subtraction of the transmissions of the transfer parameters $\tau_{11}$ and $\tau_{21}$ :

$$
V_{d}=\left|\tau_{11}\right|^{2}-\left|\tau_{21}\right|^{2}=2 \sin ^{2}(\Delta \psi / 2)-1
$$

$$
\Delta \psi_{1}=N \Delta \phi_{1}+\Delta \varphi_{1}, \Delta \psi_{2}=N \Delta \phi_{2}+\Delta \varphi_{2}, \Delta \varphi_{1}=2 \pi n_{e} L_{1} / \lambda, \Delta \varphi_{2}=2 \pi n_{e} L_{2} / \lambda
$$




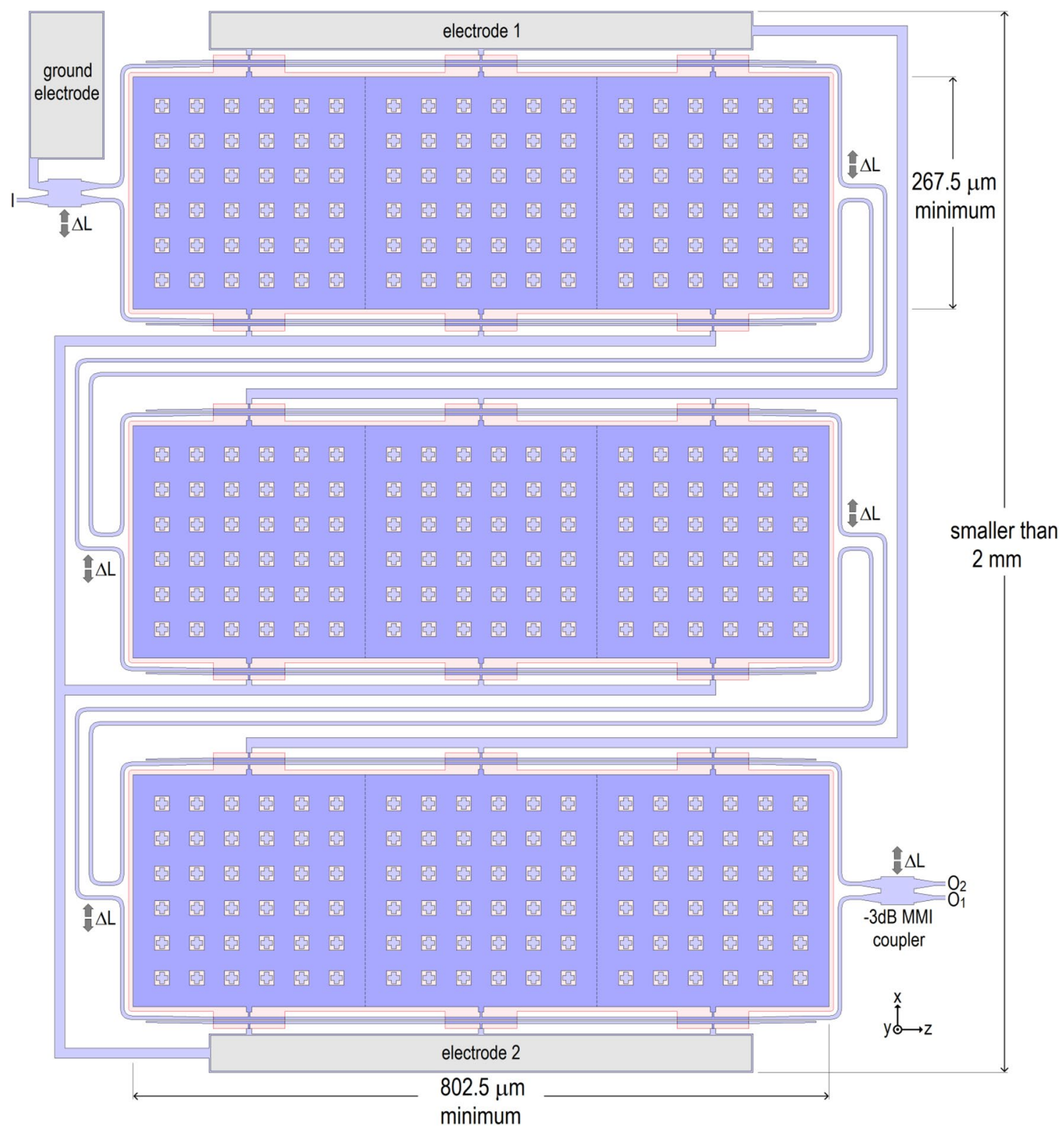

Fig. 8 Structure of the cascaded voltage-controlled adaptive-sensitivity accelerometer with increased bandwidth. Dimensions of the components in this figure are as those in Fig. 7

where Eq. (5) has been substituted in Eq. (6). The detection arithmetic unit may utilize Eq. (6) to determine the phase difference $\Delta \phi$ modulated due to the applied acceleration. The relation between the acceleration and $\Delta \phi$ for an applied common voltage $\left(V_{1}=V_{2}\right)$ is linear for the proposed device as demonstrated in the results of Sect. 4. According to Eq. (6), $\Delta \psi$ should be set to $\pm 90^{\circ}$ when the acceleration of the carrying template is zero to yield in $V_{d}=0$. Then, the polarity of $V_{d}$ indicates whether the applied acceleration is in the positive or negative directions of the $x$-axis. The operation of the detector can be set either in the broadband or periodic transmission modes.
In the broadband mode, the path length difference $\Delta L$ is either eliminated or is set to $\Delta L=\lambda \Delta \varphi /\left(2 \pi n_{e}\right) \approx \pm 180 \mathrm{~nm}$ taken for the parameters of $\lambda=1.55 \mu \mathrm{m}, \Delta \varphi= \pm 90^{\circ}$, and $n_{e} \approx 2.151$ which is simulated at $42{ }^{\circ} \mathrm{F}$ temperature. The short path length difference of $\Delta L= \pm 180 \mathrm{~nm}$ sets $\Delta \psi= \pm 90^{\circ}$ and $V_{d}=0$ over a broadband range in the $C$-band at zero acceleration providing that $V_{1}=V_{2}$. The elimination of the path length difference $(\Delta L=0)$ which yields $\Delta \varphi=0^{\circ}$ can be compensated by applying proper non-zero potential difference $\left(\Delta V=V_{1}-V_{2} \neq 0\right)$ that is required to calibrate $\Delta \psi=N \Delta \phi= \pm 90^{\circ}$ when the acceleration is zero. 
In the periodic mode, long $\Delta L$ value is used to produce sinusoidal transmission responses versus wavelength at outputs $O_{1}$ and $O_{2}$. The consecutive peaks of the periodic transmission responses of outputs $\mathrm{O}_{1}$ and $\mathrm{O}_{2}$ are separated by half the FSR wavelength which is given by $\Lambda=\lambda^{2} /\left(n_{g} \Delta L\right)$, where $n_{g} \approx 5.068$ is the group index simulated at $\lambda=1.55 \mu \mathrm{m}$ and $42^{\circ}$. The change in phase $\Delta \phi$ due to an applied acceleration or due to an applied non-zero potential difference $\Delta V \neq 0$ results in wavelength-steering the transmission responses of outputs $\mathrm{O}_{1}$ and $\mathrm{O}_{2}$. In theory, the conversion of the wavelength shift in the transmission responses of outputs $O_{1}$ and $O_{2}$ due to an applied acceleration into intensity modulation of the optical carriers that are applied to the PDs can be achieved by using a single-tone optical carrier. The wavelength of the optical signal has to be calibrated at exactly midway between two consecutive peaks of the transmission responses of outputs $\mathrm{O}_{1}$ and $\mathrm{O}_{2}$ at zero acceleration. This can be achieved by either tuning the wavelength of the optical source or by applying a proper potential difference $\Delta V$ to steer the periodic transmission responses to the desired wavelength location that matches the optical source. The transmissions of outputs $O_{1}$ and $O_{2}$ are equal at the wavelength point midway between two consecutive peaks of the two outputs. Hence, at zero acceleration the matching of the wavelength of the input optical signal with the wavelength point midway between two consecutive peaks of the transmission responses of outputs $O_{1}$ and $O_{2}$ calibrates $\Delta \psi=0$ and $V_{d}=0$ in Eq. (6).

According to Eq. (6), for both of the broadband and periodic modes, the limits for the maximum measurable acceleration are reached when $\Delta \phi$ varies within the range of $\pm 90^{\circ} / \mathrm{N}$. In conclusion, the sensitivity of the accelerometer is not affected by the chosen broadband or periodic transmission mode detection method, and the offsetting at zero acceleration is electronically possible since $\Delta \phi$ is a function of the electrode voltages.

\section{Modeling and analysis of one section of the accelerometer}

This part presents the motion analysis for one section of the opto-electromechanical accelerometer formed of a seismic mass $m$ anchored between two suspended slot waveguide voltage-controlled springs. The modeling of the opto-electromechanical accelerometer section is explained in the free body vector diagram for the distributed and point forces of Fig. 9. The voltage-controlled springs of four suspended Si beams are labeled as $1 a, 1 b$, $2 b$, and $2 a$ from top to bottom of the figure. In the absence of angular momentums, the deflection in the suspended $\mathrm{Si}$ beams in the transverse $x$-direction is symmetrical along the $z$-axis around the midpoint $z=L_{s} / 2$. The seismic mass applies acceleration point forces $f_{\delta 1}$ and $f_{\delta 2}$ to the Si beams $1 b$ and $2 b$, respectively, at their midpoints along the $z$ -axis. The mass density of each Si beam applies distributed acceleration force $q_{n}$ in the direction of motion along the $x$-axis. The potential differences $V_{1}$ and $V_{2}$ result in distributed attraction forces $q_{1 v}$ and $q_{2 v}$, respectively, acting between the capacitive plates of the inner surfaces of the Si beams of each slot waveguide. The flexural rigidity of each suspended Si beam applies balancing distributed force in reaction to modulating the excursion width $W_{e x}$ of the beam along the $z$-axis. The modulated gap widths $W_{s 1}$ and $W_{s 2}$ of the two suspended Si slot waveguide springs are related to the excursion widths according to Fig. 9 by the relations:

$W_{s 1}=W_{s 0}+W_{e \times 1 a}-W_{e \times 1 b}, W_{s 2}=W_{s 0}-W_{e \times 2 a}+W_{e \times 2 b}$

$W_{\text {ex1ar }}, W_{\text {ex1b }}, W_{\text {ex2a, }}$ and $W_{\text {ex2b }}$ are the excursion widths of beams $1 a, 1 b, 2 a$, and $2 b$, respectively. The acceleration force $f_{m}$ of the seismic mass is equal to the sum of the point forces $f_{\delta 1}$ and $f_{\delta 2}$ :

$f_{m}=m a=f_{\delta 1}+f_{\delta 2}$

The modulated phase difference $\Delta \phi$ of the accelerometer section of seismic mass $m$ is given by:

$\Delta \phi=\Delta \phi_{1}-\Delta \phi_{2}$

$\Delta \phi_{k}=\frac{2 \pi}{\lambda}\left[2 \int_{0}^{L_{s} / 2} n_{s k} d z-n_{s 0} L_{s}\right]$

$k$ in Eq. (9) is either 1 or 2 and it addresses the two differential phase shift slot waveguides. The effective index $n_{s k}$ of the phase shift element $k$ is a function of the modulated gap width $W_{s k}(z)$, and hence it depends on both the acceleration and voltages $V_{1}$ and $V_{2} \cdot n_{s 0}$ is the effective index of the phase shift element at the reset condition of zero acceleration and $V_{1}=V_{2}=0$. Due to the similarity in modulating the gap width of the suspended slot waveguide along the $z$-axis around the midpoint $z=L_{s} / 2$ only the motion response for half the length of the suspended $\mathrm{Si}$ beam is needed to be considered in the analysis. The Euler-Bernoulli $4^{\text {th }}$ order non-linear differential equations well known in the literature that describe the balanced distributed forces of the four suspended Si beams are given by:

$E I \frac{d^{4} W_{e \times 1 a}}{d z^{4}}=-q_{1 v}+q_{n}, E I \frac{d^{4} W_{e \times 1 b}}{d z^{4}}=q_{1 v}+q_{n}+\frac{f_{\delta 1}}{z_{0}} \delta\left(z-L_{s} / 2\right)$ 


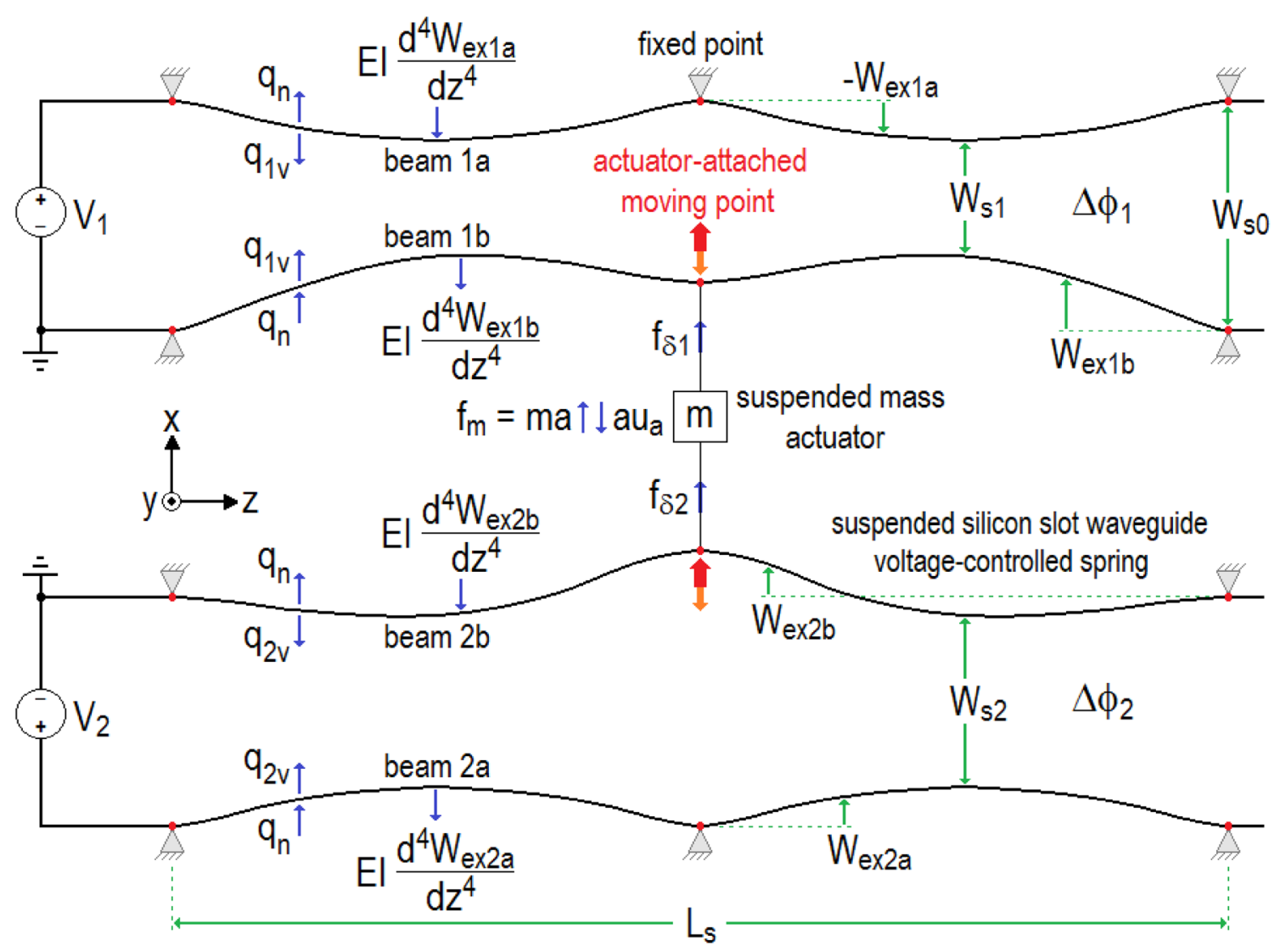

Fig. 9 Distributed force analysis for one section of the opto-electromechanical accelerometer

$E I \frac{d^{4} W_{e \times 2 a}}{d z^{4}}=q_{2 v}+q_{n}, E I \frac{d^{4} W_{e \times 2 b}}{d z^{4}}=-q_{2 v}+q_{n}+\frac{f_{\delta 2}}{z_{0}} \delta\left(z-L_{s} / 2\right)$

$E$ is Young's modulus and is equal to $169 \mathrm{GPa}$ in the direction of interest [23]. The beam area moment of inertia $I$ in the direction of interest is given in terms of the $\mathrm{Si}$ beam width $W_{g s}$ and thickness $h$ by [23]:

$I=\frac{h W_{g s}^{3}}{12}$

The distributed force $q_{n}$ due to accelerating the mass density of each suspended Si beam is given by:

$q_{n}=h W_{g s} m_{d} a$

The distributed attraction force $q_{1 v}=E_{1} / W_{s 1}$ between the inner surfaces of beams $1 a$ and $1 b$, where $E_{1}$ is the energy per unit length of the distributed capacitance $C_{1}$ formed between the inner surfaces of beams $1 a$ and $1 b$, and similarly the distributed attraction force $q_{2 v}$ between the inner surfaces of beams $2 a$ and $2 b$, are given by: $q_{1 v}=\frac{\varepsilon_{o} h}{2}\left(\frac{V_{1}}{W_{s 1}}\right)^{2}, q_{2 v}=\frac{\varepsilon_{o} h}{2}\left(\frac{V_{2}}{W_{s 2}}\right)^{2}$

where $\varepsilon_{0}$ is the free space permittivity. In Eq. (10) $\delta\left(z-L_{s} / 2\right)$ is the Dirac delta function defined in the numerical solution here as:

$\delta\left(z-L_{s} / 2\right)= \begin{cases}1 & \text { atz }=L_{s} / 2 \\ 0 & \text { otherwise }\end{cases}$

A uniform sampling pitch of $z_{o}=L_{s} / N$ may be used in the numerical procedure of Eq. (10), where $N+1$ represents the total number of uniformly spaced samples taken along the beam length $L_{s}$. Accordingly, $N$ should be always taken as an even number to account for the actuation point forces $f_{\delta 1}$ and $f_{\delta 2}$.

The numerical solution of Eq. (10) can be subjected to fixed and hinged boundary conditions at the edge clamping points of the beams (i.e. at $z=0$ and $z=L_{s}$ ). The fixed boundary conditions (FBCs) establish a more rigorous restriction on the bending flexibility of the beams, whereas the hinged boundary conditions (HBCs) allow maximum 
bending flexibility of the beams. The FBCs allow no displacement and no rotation at the clamping points:

$\left.\begin{array}{l}W_{e \times 1 a}=W_{e \times 1 b}=W_{e \times 2 a}=W_{e \times 2 b}=0 \\ \frac{d W_{e \times 1 a}}{d z}=\frac{d W_{e \times 1 b}}{d z}=\frac{d W_{\text {ex2a }}}{d z}=\frac{d W_{e \times 2 b}}{d z}=0\end{array}\right\}$ atz $=0$ and $z=L_{s}$

The HBCs allow no displacement, but they allow rotation at the clamping points:

$\left.\begin{array}{c}W_{e \times 1 a}=W_{e \times 1 b}=W_{e \times 2 a}=W_{e \times 2 b}=0 \\ \frac{d^{2} W_{e \times 1 a}}{d z^{2}}=\frac{d^{2} W_{\text {ex1b }}}{d z^{2}}=\frac{d^{2} W_{\text {ex2a }}}{d z^{2}}=\frac{d^{2} W_{\text {ex2b }}}{d z^{2}}=0\end{array}\right\} a t z=0$ and $z=L_{s}$

The even symmetry in the excursion width response of the four beams along the $z$-axis around the midpoint $z=L_{s} / 2$ establishes the following set of symmetry conditions taken for the range of $z$ from 0 to $L_{s} / 2$ :

$\left.\begin{array}{l}W_{\text {ex1a }}(z)=W_{\text {ex1a }}\left(L_{s}-z\right), W_{\text {ex1b }}(z)=W_{\text {ex1b }}\left(L_{s}-z\right) \\ W_{\text {ex2a }}(z)=W_{\text {ex2a }}\left(L_{s}-z\right), W_{\text {ex } b}(z)=W_{\text {ex } 2 b}\left(L_{s}-z\right)\end{array}\right\}$ for $0<z<\frac{L_{s}}{2}$

The mid-length points of beams $1 a$ and $2 a$ are fixed in position by the voltage-feeding studs shown in Fig. 7. This establishes the following additional boundary condition:

$W_{\text {ex1a }}\left(L_{s} / 2\right)=W_{\text {ex2a }}\left(L_{s} / 2\right)=0$

The mid-length points of beams $1 b$ and $2 b$ are attached to the actuator mass, and hence they move together laterally along the $x$-axis. This establishes the following last boundary condition:

$W_{e x 1 b}\left(L_{s} / 2\right)=W_{e \times 2 b}\left(L_{s} / 2\right)$

Equations (7) through (19) establish the full mathematical model used to solve Eq. (10) numerically subjected to the stated set of boundary and symmetry conditions to determine the excursion widths of the four suspended Si beams and the phase shift introduced by the phase shift element utilizing the simulation of the effective index of the slot waveguide as a function of the modulated gap width. The simulation of the effective index of the slot waveguide taken for the fundamental mode as a function of the gap width is depicted in Fig. 10 for the range of $W_{s}$ of 20-180 $\mathrm{nm}$ which represents the maximum dynamic range for modulating the gap width. The simulations in this figure achieved utilizing the mode designer of the VPIphotonics industrial simulation environment are taken for the Si base layer thicknesses of $300 \mathrm{~nm}$ and $220 \mathrm{~nm}$ with the parameters of $W_{g s}=230 \mathrm{~nm}$ and $42 \mathrm{oF}$ temperature. The results in this figure are found in agreement with those simulated in [25] utilizing the FIMMPROP tool from Photon Design that was taken for the range of $W_{s}$ of 20-120 $\mathrm{nm}$ and those simulated in [23]. The

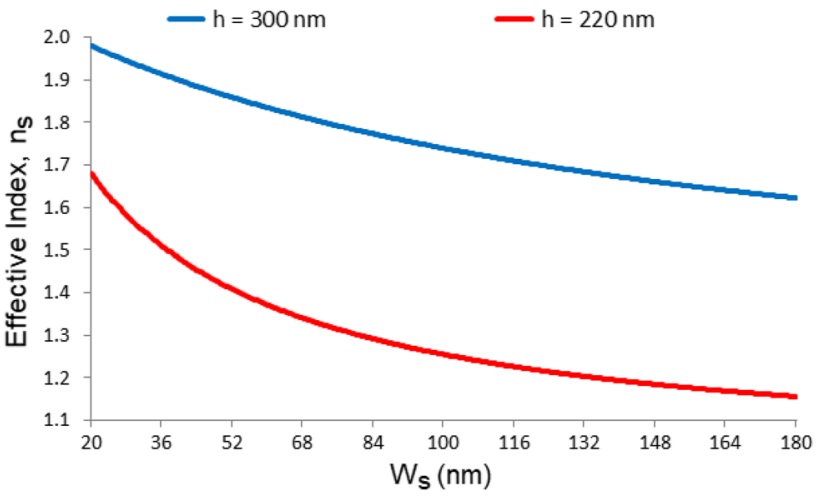

Fig. 10 Effective index $n_{s}$ of the slot waveguide as a function of the slot width $W_{s}$ simulated for $W_{g s}=230 \mathrm{~nm}$ and $42{ }^{\circ} \mathrm{F}$

simulation of the effective index of Fig. 10 is found to have a weak positive temperature coefficient dependence of around $\Delta n_{s}=1.2071 \times 10^{-4}\left({ }^{\circ} \mathrm{F}\right)^{-1}$ for $h=300 \mathrm{~nm}$ and $\Delta n_{s}=3.5532 \times 10^{-5}\left({ }^{\circ} \mathrm{F}\right)^{-1}$ for $h=220 \mathrm{~nm}$. Since the ratio of the suspended Si beam length to the maximum possible excursion width is very large the curvature radius of the suspended Si beam is very large and hence it is expected that the stress-induced birefringence effect [52] on modulating the effective index of the slot waveguide to be minimal in comparison to the change of the refractive index due to modulating the slot width. The numerical solution of the mathematical model presented in this section and the simulation of the effective index of Fig. 10 are both used in the next section for studying the characteristics and behavior of the proposed accelerometer.

\section{Characteristics study of the opto-electromechanical accelerometer}

The performance of the proposed opto-electromechanical accelerometer is studied in this section based on the numerical approach of the previous section. A smaller test mass requires the use of longer suspended $\mathrm{Si}$ beams and vice-versa to incur the same amount of accelerationdependent phase shift differences. On the other hand, taking a larger test mass would result in a larger footprint of the structure and vice-versa. A test mass of $m=50 \mathrm{ng}$ is taken as a study example in all consequent simulation results.

The simulation of the phase differences $\Delta \phi_{1}$ and $\Delta \phi_{2}$ as functions of the slot waveguide length $L_{s}$ taken for both FBCs and HBCs at an acceleration of $a=100 \mathrm{~ms}^{-2}$ are depicted in Fig. 11. The electrode voltages are taken at reset in this simulation (i.e. $V_{1}=V_{2}=0$ ) to yield 


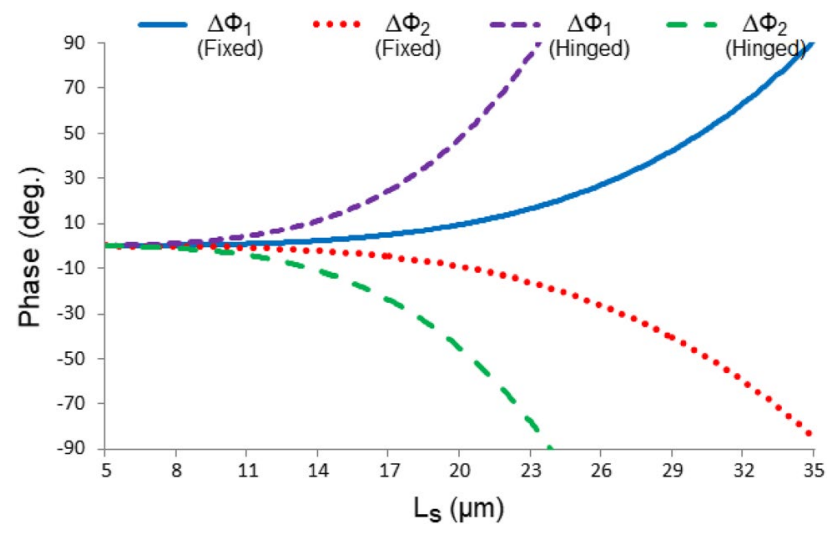

Fig. 11 The phase differences $\Delta \phi_{1}$ and $\Delta \phi_{2}$ versus the design length $L_{s}$ for FBCs and HBCs. "Fixed" and "Hinged" in the legend refer to FBCs and HBCs, respectively. $m=50 \mathrm{ng}, a=100 \mathrm{~ms}^{-2}$, and $V_{1}=V_{2}=0$

symmetrical characteristics of $\Delta \phi_{1}$ and $\Delta \phi_{2}$. The characteristics of this figure can be used to decide on the length $L_{s}$ required to achieve a phase shift difference $\Delta \phi$ based on the number of cascaded accelerometer sections taken for the detection of $100 \mathrm{~ms}^{-2}$ of maximum acceleration at reset voltages. As an example, for two cascaded accelerometer sections $(N=2)$ achieving $\Delta \phi=90^{\circ} / N=45^{\circ}$ requires the use of $L_{s}=25 \mu \mathrm{m}$ based on FBCs. This length of the suspension beams is taken as a study case in many consequent simulations. Increasing the number of cascaded accelerometer sections to $N=9$ as per the system of Fig. 8 requires $\Delta \phi=10^{\circ}$ which can be achieved at $L_{s}=17 \mu \mathrm{m}$ based on FBCs. Reducing the length $L_{s}$ which requires increasing the number of cascaded sections of the accelerometer has the benefit of increasing the operation bandwidth. The redrawing of the characteristic of Fig. 11 for higher maximum measurable acceleration at reset voltages yields in smaller values of the length $L_{s}$ allowing increasing the operation speed.

The simulation of the design length $L_{s}$ as a function of the logarithm of the maximum measurable acceleration (i.e. $\left.\log \left(a_{m}\right)\right)$ at reset voltages for both FBCs and HBCs is depicted in Fig. 12 taking $\Delta \phi$ as a parameter. The characteristics of this figure can be used to predict the length $L_{s}$ required for maximum measurable acceleration at reset voltages for a given number of cascaded sections. A different $\Delta \phi$ value used in the legend of this figure corresponds to the use of a different number of sections. The $\Delta \phi$ values of $45^{\circ}$ and $10^{\circ}$ match the use of two and nine cascaded sections, respectively. It is observed that a length of $L_{s} \approx 5.4 \mu \mathrm{m}$ based on FBCs is required for maximum measurable acceleration of $a=10^{4} \mathrm{~ms}^{-2}$ at reset voltages when nine cascaded sections $(N=9)$ are used as per the system of Fig. 8 to achieve up to $\pm 90^{\circ}$ of total phase shift. This short length is used to investigate the maximum

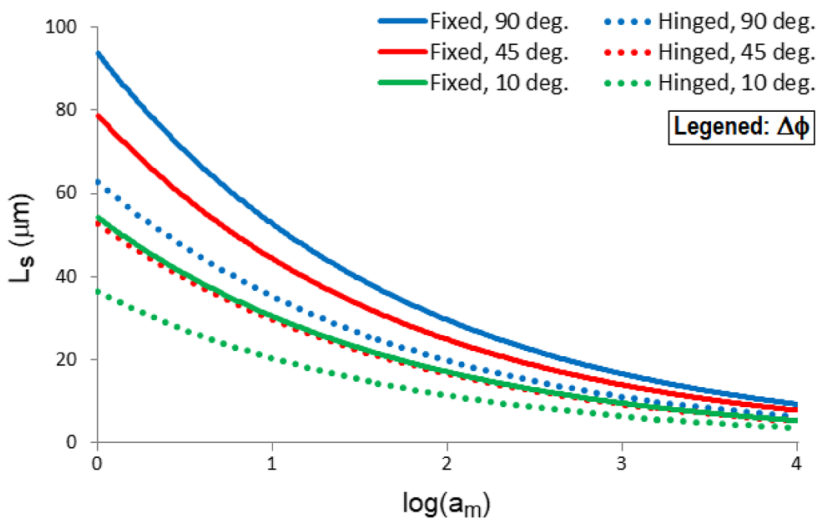

Fig. 12 The design length $L_{s}$ versus $\log \left(a_{m}\right)$ at reset voltages for FBCs and HBCs taken for different $\Delta \phi$ values as indicated in the legend. "Fixed" and "Hinged" in the legend refer to FBCs and HBCs, respectively. $m=50 n g$ and $V_{1}=V_{2}=0$

bandwidth that may be achieved based on standard foundries.

The equivalent spring constant $k$ of one section of the sensor is a reflection of its suspension system rigidity. A higher value of $k$ makes the suspension system more rigid and it makes the sensor less sensitive to acceleration changes. The spring constant $k$ is simulated in Fig. 13 as a function of the design length $L_{s}$ of the suspended $\mathrm{Si}$ beams based on FBCs taking $a=100 \mathrm{~ms}^{-2}$ and $V_{1}=V_{2}=0$. This simulation can help in design to decide on the useful range of the length $L_{s}$. Taken at reset voltages, a smaller $k$ value obtained at a larger $L_{s}$ value increases the sensitivity of the accelerometer, whereas a larger $k$ value obtained at a smaller $L_{s}$ value increases the bandwidth response of the accelerometer. It is possible to look into optimizing the design between the requirements of achieving high sensitivity and wide bandwidth response of the sensor.

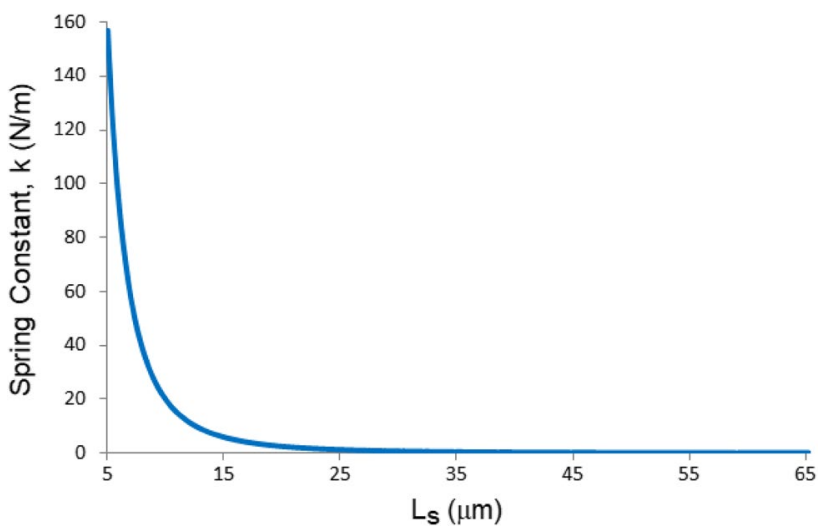

Fig. 13 The equivalent spring constant $k$ versus the design length $L_{s}$ of the suspended slot waveguide at reset voltages based on FBCs. $m=50 n g, a=100 \mathrm{~ms}^{-2}$, and $V_{1}=V_{2}=0$ 
The knee point of the $k$ characteristic curve of this figure may be eligible in design.

The fundamental frequency of resonance $f_{n}$ of the suspension system indicates the speed of detecting timevarying accelerations. The estimation of $f_{n}$ can be based on the deflection in the seismic mass $x$ determined for an applied acceleration $a$ and electrode voltages $V_{1}$ and $V_{2}$ using the relation:

$f_{n}=\frac{1}{2 \pi} \sqrt{\frac{a}{x}}$

Figure 14 depicts the simulation of $f_{n}$ based on FBCs as a function of the design length $L_{s}$ taking $V_{1}=V_{2}=0$ and $a=100 \mathrm{~ms}^{-2}$. Based on the simulated spring constant $k$ of Fig. 13 the design might be carried at the knee point of the $k$-curve to optimize between achieving high sensitivity and wide bandwidth response of the

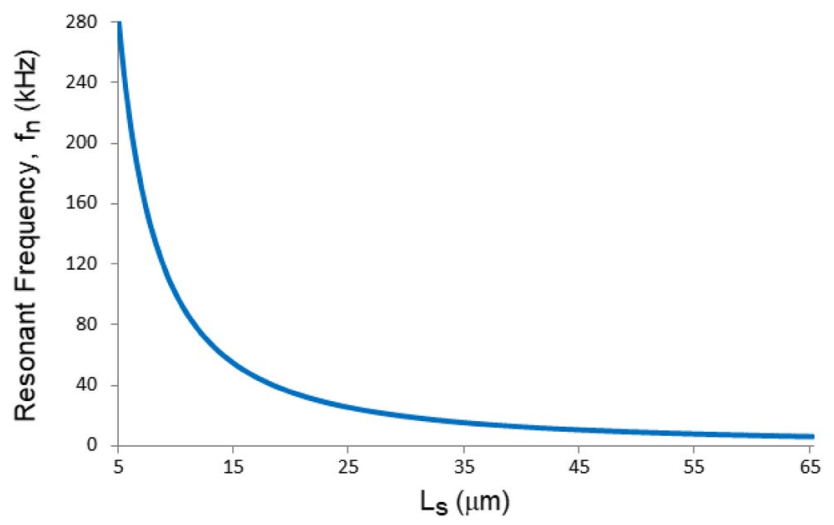

Fig. 14 The fundamental frequency of resonance of the suspension system $f_{n}$ versus the design length $L_{s}$ of the suspended slot waveguide at reset voltages based on FBCs. $m=50 n g, a=100 \mathrm{~ms}^{-2}$, and $V_{1}=V_{2}=0$ accelerometer. The knee point of the $k$-curve is located at the length $L_{s}=10 \mu \mathrm{m}$ for which case the spring constant is $k=20 \mathrm{Nm}^{-1}$ and the available bandwidth based on the simulation of Fig. 14 is $100 \mathrm{kHz}$. At the design length $L_{s}=25 \mu \mathrm{m}$, which is taken for many simulations in this investigation, the available bandwidth is about $25.2 \mathrm{kHz}$. At the shortest usable length of the suspended slot waveguide indicated before of $L_{s}=5.4 \mu \mathrm{m}$ the bandwidth may reach a maximum value of about $250 \mathrm{kHz}$.

Figure 15 demonstrates the excursions of the four suspended Si beams based on FBCs when applying an acceleration of $a=150 \mathrm{~ms}^{-2}$ and zero electrode voltages $\left(V_{1}=V_{2}=0\right)$ for a suspended Si beam length of $L_{s}=25 \mu \mathrm{m}$. In this case, the excursions of beams $1 b$ and $2 b$ are similar, whereas both beams $1 a$ and $2 a$ stay at the rest position since zero voltages are applied to the electrodes. The deflection in the seismic mass, in this case, is about $x=6 \mathrm{~nm}$. In the right side part of this figure, the excursions of beams $1 a, 1 b, 2 b$, and $2 a$ are relocated around $150 \mathrm{~nm}$, $50 \mathrm{~nm},-50 \mathrm{~nm}$, and $-150 \mathrm{~nm}$, respectively, on the $x$-axis to illustrate their relative lateral deformations in reference to the rest slot width of $100 \mathrm{~nm}$. The acceleration in this example is taken over the maximum measurable range at reset voltages for two cascaded sections to increase the deformations in the beams.

The behavior of the sensor can be programmed by the electrostatic forces between the beams of the suspension slot waveguides. The characteristics of the sensor including the spring constant, sensitivity, maximum measurable acceleration, and bandwidth response are controlled by the electrode voltages $V_{1}$ and $V_{2}$. As explained in the following simulations this allows increasing the sensitivity of the sensor and expanding the dynamic range for acceleration measurement much far beyond the natural limits without applying voltages. The application of two voltage types is considered in this investigation; the common voltage
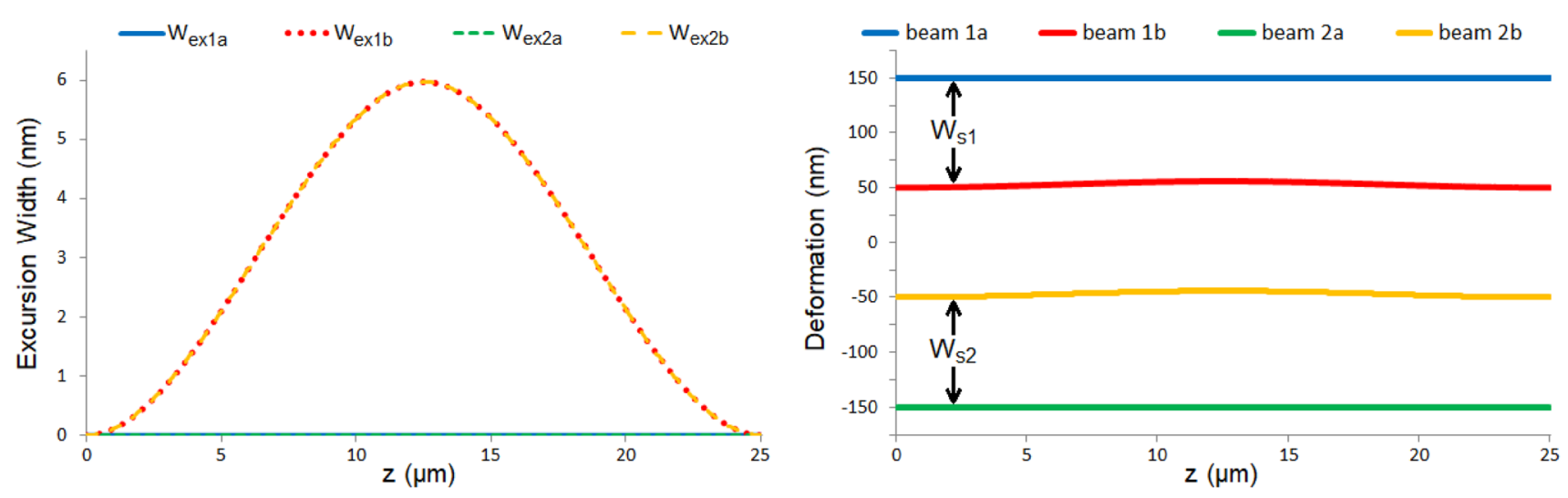

Fig. 15 Excursions of the four suspended Si beams based on FBCs. The right side part of the figure illustrates the relative deformations of the beams in the $x$-direction. $m=50 n g, L_{s}=25 \mu m, a=150 \mathrm{~ms}^{-2}$, and $V_{1}=V_{2}=0$ 
$V_{c}=\left(V_{1}+V_{2}\right) / 2$ and difference voltage $V_{d}=V_{1}-V_{2}$. At zero difference voltage $\left(V_{d}=0\right)$ the two electrode voltages are equal to the common voltage $\left(V_{c}=V_{1}=V_{2}\right)$. The application of a non-zero common voltage at zero difference voltage allows increasing the sensor sensitivity. In the negative feedback regime, the difference voltage is intended to keep the seismic mass at close distances from the standstill position. This effect allows expanding the maximum measurable acceleration over the limit without applying negative feedback by rich multiple times.

The application of an equal voltage to the electrodes of $V_{c}=V_{1}=V_{2}$ tensions the spring beams. This reduces the equivalent spring constant $k$ resulting in increasing the deflection in the seismic mass for an applied acceleration and therefore the accelerometer sensitivity is increased accordingly. Figure 16 depicts the simulation of the spring constant $k$ based on FBCs versus the common voltage $V_{c}$ taken for the design length $L_{s}=25 \mu \mathrm{m}$ and zero difference voltage. The spring constant in this simulation is reduced within a wide range from $1.256 \mathrm{~N} / \mathrm{m}$ to $0.0671 \mathrm{~N} / \mathrm{m}$ when $V_{c}$ is increased from $0 \mathrm{~V}$ to $10.1 \mathrm{~V}$. The dynamic range for controlling the spring constant in this case is about $25.4 \mathrm{~dB}$. Simulation results show that much increase in $V_{c}$ above $10.1 \mathrm{~V}$ may subject the beams to the pull-in effect and the sensor may become unstable due to the high sensitivity.

Figure 17 depicts the deflection $x$ in the seismic mass based on $\mathrm{FBCs}$ versus acceleration taking the common voltage $V_{c}$ as a parameter at zero difference voltage and using $L_{s}=25 \mu \mathrm{m}$. The conclusion that can be drawn from this figure is that the deflection in the seismic mass increases rapidly with the increase in the applied common voltage for the same acceleration. This is due to the dependence of the electrostatic force on the square of the voltage in Eq. (13). For this reason, increasing the common voltage above a critical value may cause the suspended

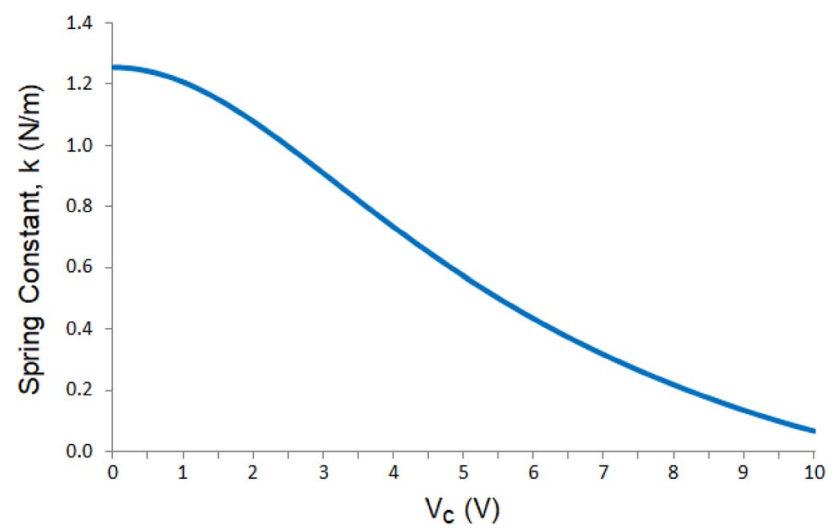

Fig. 16 Equivalent spring constant $k$ versus the common voltage $V_{c}$ based on FBCs. $m=50 \mathrm{ng}, L_{s}=25 \mu m$, and $V_{1}=V_{2}$

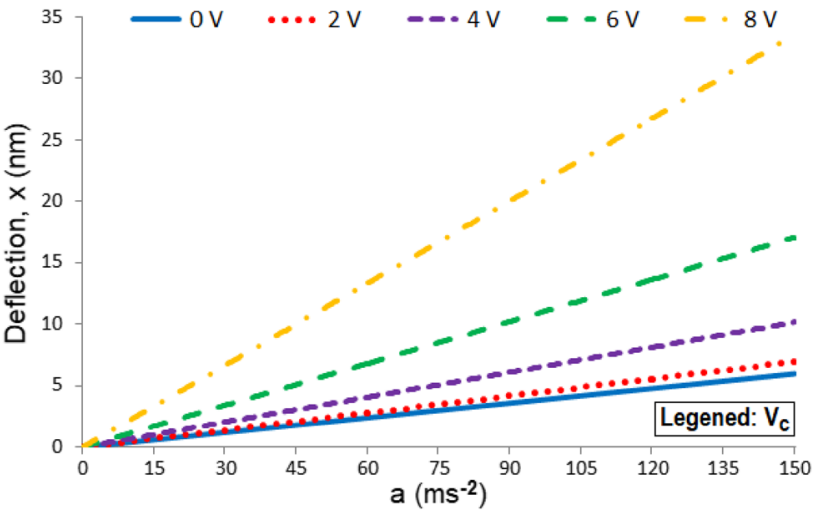

Fig. 17 Deflection $x$ in the seismic mass based on FBCs versus acceleration taking the common voltage $V_{c}$ as the legend. $m=50 n g, L_{s}=25 \mu m$, and $V_{1}=V_{2}$

beams of the slot waveguide to be pulled in until their inner surfaces become in direct contact with each other. In such a case adhesion and bonding of the movable parts may cause possible failure to the structure. For equal electrode voltages within the controllable sensitivity range, the deflection in the seismic mass increases linearly with the increase in acceleration as in the figure.

Figure 18 shows the excursions of the four suspended $\mathrm{Si}$ beams of length $L_{s}=25 \mu \mathrm{m}$ based on FBCs when applying an acceleration of $a=150 \mathrm{~ms}^{-2}$ as was the case in Fig. 15 but with the application of $V_{c}=8 \mathrm{~V}$ at zero difference voltage to strongly boost the sensitivity of the accelerometer. The right side part of this figure illustrates the relative lateral deformations of the beams in the $x$-direction by displacing the excursions of the beams by $100 \mathrm{~nm}$ distances from each other as before. In this simulation, the deformations of the beams are made excessive on purpose to make them appear clear due to applying high acceleration much beyond the maximum measurable limit for a sensor that is set extra sensitive by applying high equal electrode voltages. The $z$-dependant distributed excursions of beams $1 b$ and $2 b$ are different from each other except at their mid-length points that are attached to the seismic mass because the distributed electrostatic attraction force between beams $1 a$ and $1 b$ is different than that between beams $2 a$ and $2 b$ due to the applied non-zero acceleration. This is because when the seismic mass moves in the $x$ -axis direction due to the applied acceleration then beams $1 a$ and $1 b$ become closer to each other compared to the modulated gap width between beams $2 a$ and $2 b$ according to Eqs. (14) and (15). The $z$-dependant distributed excursions of beams $1 a$ and $2 a$ are also different from each other for the same reason. The deflection in the seismic mass, in this case, is increased to $x=33.6 \mathrm{~nm}$ compared to that of Fig. 15 of $6 \mathrm{~nm}$ due to the same applied acceleration reflecting the fact of increasing the mechanical sensitivity 

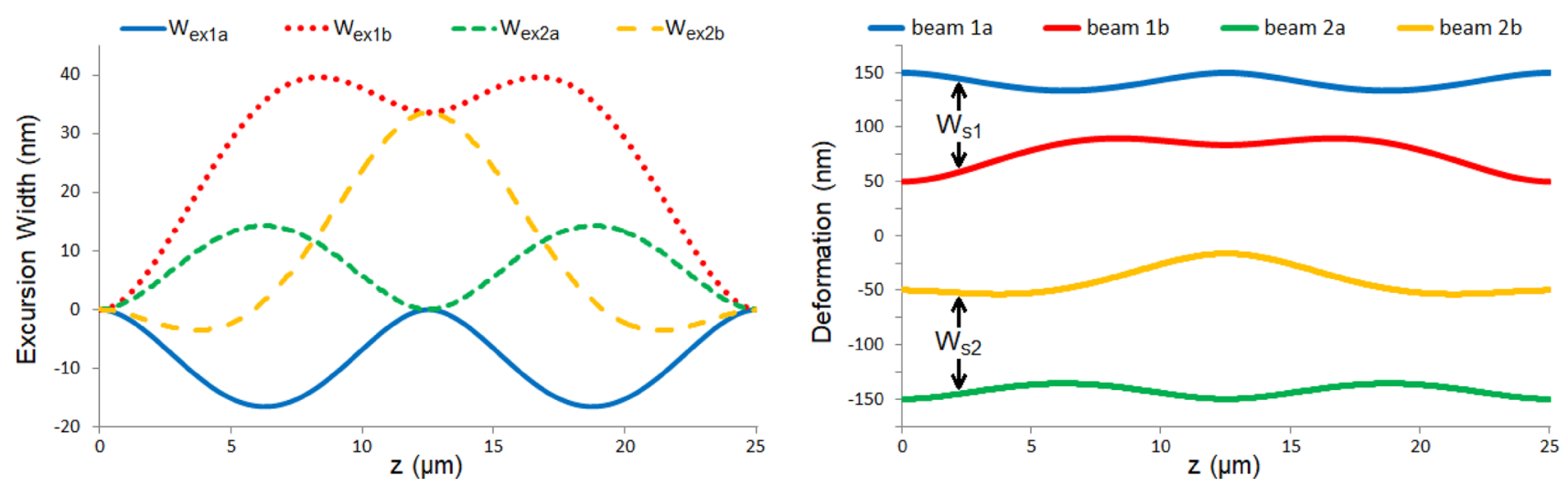

Fig. 18 Excursions of the four suspended Si beams based on FBCs. The right side part of the figure illustrates the relative deformations of the beams in the $x$-direction. $m=50 \mathrm{ng}, L_{s}=25 \mu \mathrm{m}, a=150 \mathrm{~ms}^{-2}$, and $V_{1}=V_{2}=8 \mathrm{~V}$

of the accelerometer due to the applied equal electrode voltages. The extra reduction in the distributed gap width between beams $1 a$ and $1 b$ that are getting closer to each other compared to that between beams $2 a$ and $2 b$ when a non-zero acceleration is applied also reflects the fact of increasing the optical sensitivity of the accelerometer. The distributed electrostatic force between beams $1 a$ and $1 b$ increases as these two beams get closer to each other whereas the distributed electrostatic force between beams $2 a$ and $2 b$ decreases as these two beams get farther from each other when applying a non-zero acceleration. Therefore, the equal electrode voltages further pull the seismic mass in its motion direction due to the imbalance in the distributed electrostatic force between beams $1 a$ and $1 b$ and the distributed electrostatic force between beams $2 a$ and $2 b$ incurred due to the applied acceleration. Having beams $1 a$ and $1 b$ getting closer to each other than beams $2 a$ and $2 b$ due to the imbalance in the electrostatic forces, and noting that the modulated gap width between beams $1 a$ and $1 b$ is getting smaller midway between the edge suspension points of the beams and the suspension point of the seismic mass, enhances modulating the phase difference $\Delta \phi$. This effect increases the dynamic range of the optical sensitivity more than it increases the dynamic range of the mechanical sensitivity of the sensor.

The wide dynamic range for controlling the optical sensitivity of the accelerometer is explained in Fig. 19. In this figure, the phase difference $\Delta \phi$ is simulated based on FBCs as a function of acceleration taking $V_{c}$ as a parameter at zero difference voltage. The length of the suspended slot waveguide is taken as $L_{s}=25 \mu \mathrm{m}$ as before. Increasing $V_{c}$ at zero difference voltage can increase the optical sensitivity greatly as seen in the figure. As an example, to achieve $\Delta \phi=45^{\circ}$ for two cascaded sections the maximum measurable acceleration is about $98.4 \mathrm{~ms}^{-2}$ when $V_{c}=V_{d}=0$. However, when $V_{c}$ is increased to $10 \mathrm{~V}$ at

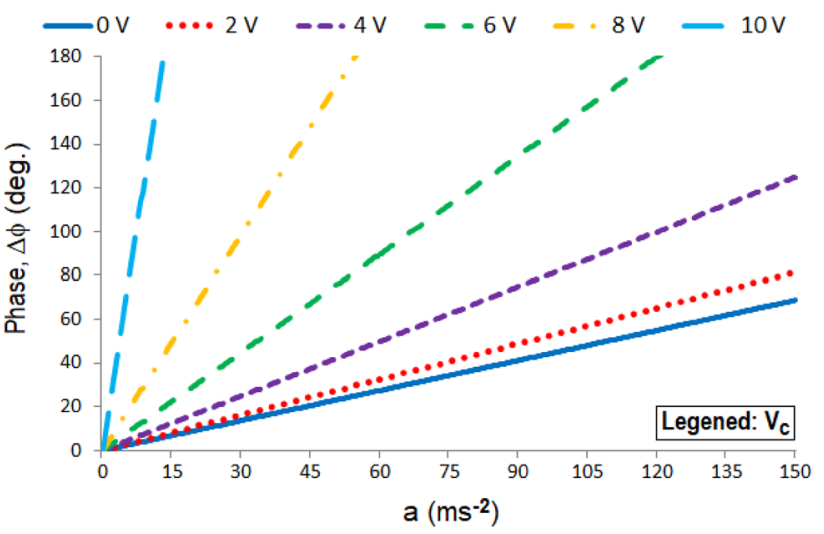

Fig. 19 The phase difference $\Delta \phi$ versus acceleration based on FBCs taking the common voltage $V_{c}$ as the legend. $m=50 \mathrm{ng}, L_{s}=25 \mu \mathrm{m}$, and $V_{1}=V_{2}$

$V_{d}=0$, the maximum measurable acceleration is reduced to about $3.32 \mathrm{~ms}^{-2}$. This major increase in optical sensitivity allows efficient sensing of tiny sub-gravitational accelerations. The simulation in this figure also shows that the phase shift $\Delta \phi$ is a linear function of acceleration for equal electrode voltages.

In a traditional single section optical sensor, the mechanical sensitivity $(\Delta x / \Delta a)$ indicates the distance shift $\Delta x$ in the seismic mass in response to a change in acceleration $\Delta a$, and the optical sensitivity $(\Delta P / \Delta a)$ indicates the change in the detection optical power $\Delta P$ in response to a change in acceleration $\Delta a$. In the multi-section architectures of Figs. 6 and 8, the mechanical and optical sensitivities of one section are multiplied by the number of sections $N$ used in the sensor to obtain the total effective sensitivities. The simulations of the mechanical and optical sensitivities of the sensor for the length $L_{s}=25 \mu \mathrm{m}$ of the suspended slot waveguide as functions of the common voltage $V_{c}$ at zero difference voltage based on FBCs and 
taking the number of cascaded sections $N$ as a parameter are depicted in Fig. 20. In these simulations, the dynamic ranges for controlling the mechanical and optical sensitivities are $25.45 \mathrm{~dB}$ and $30.42 \mathrm{~dB}$, respectively. This confirms the statement made before that the dynamic range of the optical sensitivity is bigger than that of the mechanical sensitivity both controlled by applying equal electrode voltages. When nine cascaded sections are used with the design length $L_{s}=25 \mu \mathrm{m}$ the optical sensitivity of the sensor may be controlled by the common equal electrode voltages within the range of $44.88-1489.8 \% / g$ as in the right part of Fig. 20. Additional to the wide dynamic range for controlling the optical sensitivity of $30.42 \mathrm{~dB}$ the maximum obtainable optical sensitivity of $1489.8 \% / \mathrm{g}$ indicated here based on simulation is a hard number that is believed not reported before for at least in the other optical sensors surveyed in this study.

The application of a non-zero common voltage $V_{c}$ at zero difference voltage increases the accelerometer sensitivity for the detection of small accelerations, but it also results in decreasing the bandwidth response of the sensor. The effect of increasing $V_{c}$ at zero difference voltage on reducing $f_{n}$ is studied in Fig. 21 based on FBCs and taken for the design length $L_{s}=25 \mu \mathrm{m}$. In this simulation as $V_{c}$ is increased within the controllable range of $0-10.1 \mathrm{~V}$ the fundamental resonant frequency is decreased from about $25.2 \mathrm{kHz}$ down to about $5.83 \mathrm{kHz}$. It is concluded that the reported extreme optical sensitivity of $1489.8 \% / \mathrm{g}$ is paid for lowering the bandwidth response down to $5.83 \mathrm{kHz}$ although this value is still higher than the bandwidth response of many of the sensors listed in Table 1.

The maximum measurable acceleration $a_{m}$ is such that the phase difference $\Delta \phi$ produced by one section of the sensor should not exceed the range of $\pm 90^{\circ} / \mathrm{N}$. For a constant length $L_{s}$ of the suspended slot waveguide the increase of the number of cascaded sections $N$ used in the sensor and the increase of the common voltage $V_{c}$ to

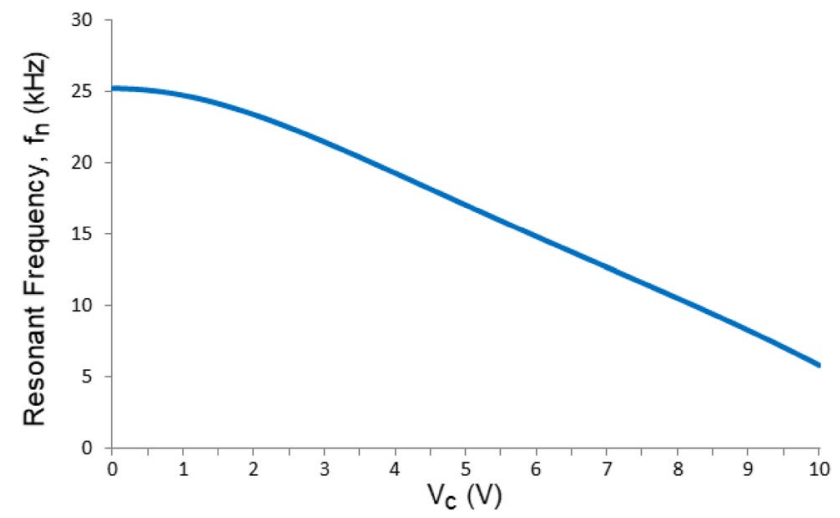

Fig. 21 The fundamental frequency of resonance of the suspension system $f_{n}$ versus the common voltage $V_{c}$ based on FBCs. $m=50 n g$, $L_{s}=25 \mu m$, and $V_{1}=V_{2}$

increase the optical sensitivity of the sensor lowers the maximum acceleration that can be measured. Figure 22 depicts the simulation of $a_{m}$ versus $V_{c}$ at $V_{d}=0$ for the case of $L_{s}=25 \mu \mathrm{m}$ and taking $N$ as a parameter. As appeared in this figure the value of $a_{m}$ decreases from about $98.32 \mathrm{~ms}^{-2}$ to about $21.85 \mathrm{~ms}^{-2}$ at reset voltages when $N$ is increased from two to nine sections. To increase $a_{m}$ at reset voltages for the nine sections, one may adjust the length $L_{s}$ for a smaller value as per the characteristics of Fig. 12 but this also results in lowering the optical sensitivity of the sensor. As a design compromise, increasing the number of cascaded sections to the maximum limit that the integration chip patterning area allows and decreasing the length of the suspended slot waveguides helps to maintain high sensitivity levels and increase the bandwidth response of the sensor.

In the negative feedback closed-loop operation of the sensor a non-zero difference voltage $V_{d}$ is applied such that to keep the detection optical power represented by the detection voltage in Eq. (6) at null values. This is
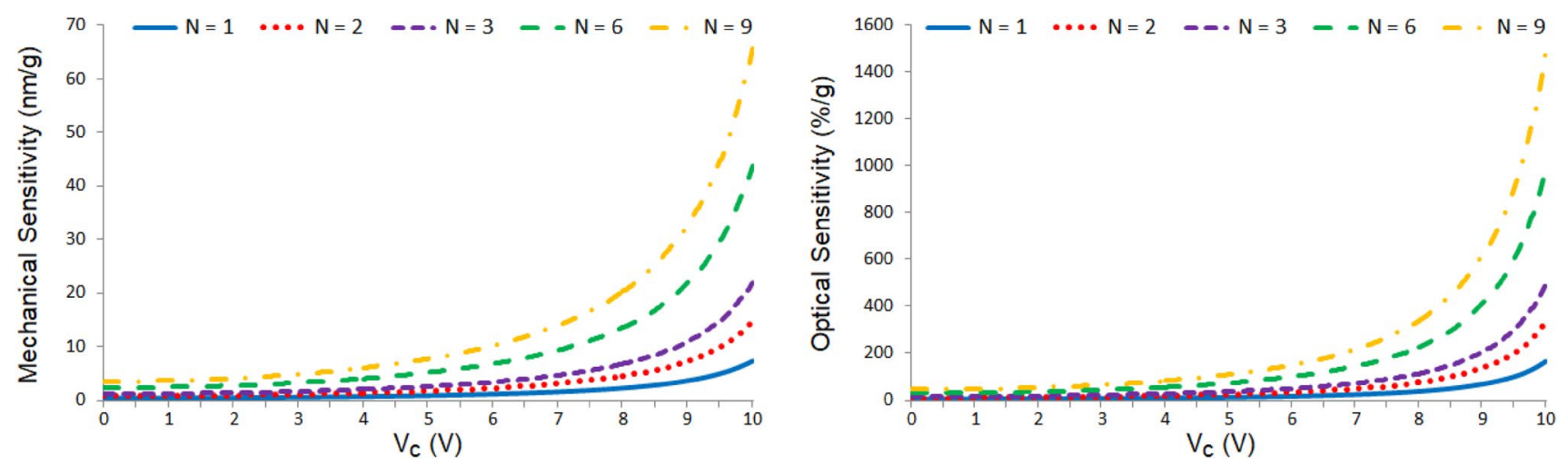

Fig. 20 The mechanical sensitivity (left) and optical sensitivity (right) versus the common voltage $V_{c}$ taking the number of cascaded sections $N$ of the sensor as the legend. $m=50 n g, L_{s}=25 \mu m$, and $V_{1}=V_{2}$ 


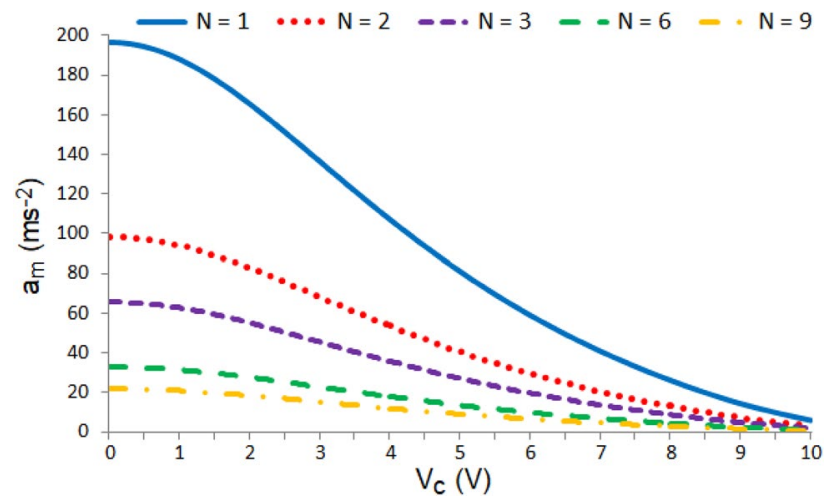

Fig. 22 The maximum measurable acceleration $a_{m}$ based on FBCs versus the common voltage $V_{c}$ taking the number of cascaded sections $N$ of the sensor as the legend. $m=50 n g, L_{s}=25 \mu m$, and $V_{1}=V_{2}$

equivalent to maintaining $\Delta \phi \approx 0$ for all applied accelerations. The distributed electrostatic force between the beams of the suspended slot waveguides in the negative feedback regime wrestles against the acceleration force to keep the seismic mass at close distances from the rest position. In this case $V_{d}$ provides a measure for the acceleration. In negative feedback control, the sensitivity of the accelerometer is reduced a lot such that the maximum measurable acceleration is gratefully increased above the natural limit at reset voltages. The sensitivity and acceleration range in the negative feedback regime are not affected by the number of cascaded sections used in the sensor. This provides relief to the maximum measurable acceleration when a large number of cascaded sections are used to provide high sensitivity figures in the openloop voltage control regime. The electrode voltages $V_{1}$ and $V_{2}$ are always positive and they cause attraction distributed forces between the beams of the slot waveguides. In negative feedback control $V_{d}$ becomes negative when the seismic mass is accelerated in the positive $x$-direction, and vice versa. Therefore, the value and polarity of $V_{d}$ indicate the value and direction of acceleration.

The simulations of the resting voltages $V_{1}, V_{2}, V_{c}$, and $V_{d}$ versus acceleration in the negative feedback regime for the length $L_{s}=25 \mu \mathrm{m}$ are demonstrated in Fig. 23 for two distinct control algorithms. In the left part of the figure $V_{1}$ is kept at reset while increasing $V_{2}$ with the increase of acceleration of the seismic mass in the positive $x$-direction. When the seismic mass is accelerated in the negative $x$-direction $V_{2}$ is kept at reset and $V_{1}$ is increased. As a result of this control scheme both $V_{c}$ and $\left|V_{d}\right|$ increase in a non-linear manner with the increase of acceleration in both positive and negative directions of the $x$-axis. In the right part of the figure $V_{c}$ is intentionally fixed at $5 \mathrm{~V}$ which is about half the maximum electrode voltage that may be applied safely without experiencing the pull-in effect of the suspended beams of the slot waveguides. In this control fashion the voltages $V_{1}, V_{2}$, and $V_{d}$ become almost linear functions of acceleration as in the figure. The simulation results for both negative feedback schemes in this figure indicate the expansion of the maximum measurable acceleration before experiencing the pull-in effect of the suspended beams for up to at least $4100 \mathrm{~ms}^{-2}$. This represents an increase in the natural limit of the maximum measurable acceleration at reset voltages of $98.32 \mathrm{~ms}^{-2}$ for two cascaded sections by a factor of 41.7 or equivalently $4070 \%$ of the increase. The smallest optical sensitivity in Fig. 20 without applying negative feedback is about $9.974 \% / g$ taken for $V_{c}=V_{d}=0$ and two cascade sections. The constant optical sensitivity in the feedback regime of the right part of Fig. 23 is about $0.239 \% / \mathrm{g}$ which is smaller than the smallest value without utilizing negative feedback. The combined dynamic range of each of the maximum measurable acceleration and optical sensitivity utilizing both open-loop and closed-loop voltage control algorithms taken for nine cascaded sections of the sensor and $L_{s}=25 \mu \mathrm{m}$ is about $76 \mathrm{~dB}$. These high sensitivities
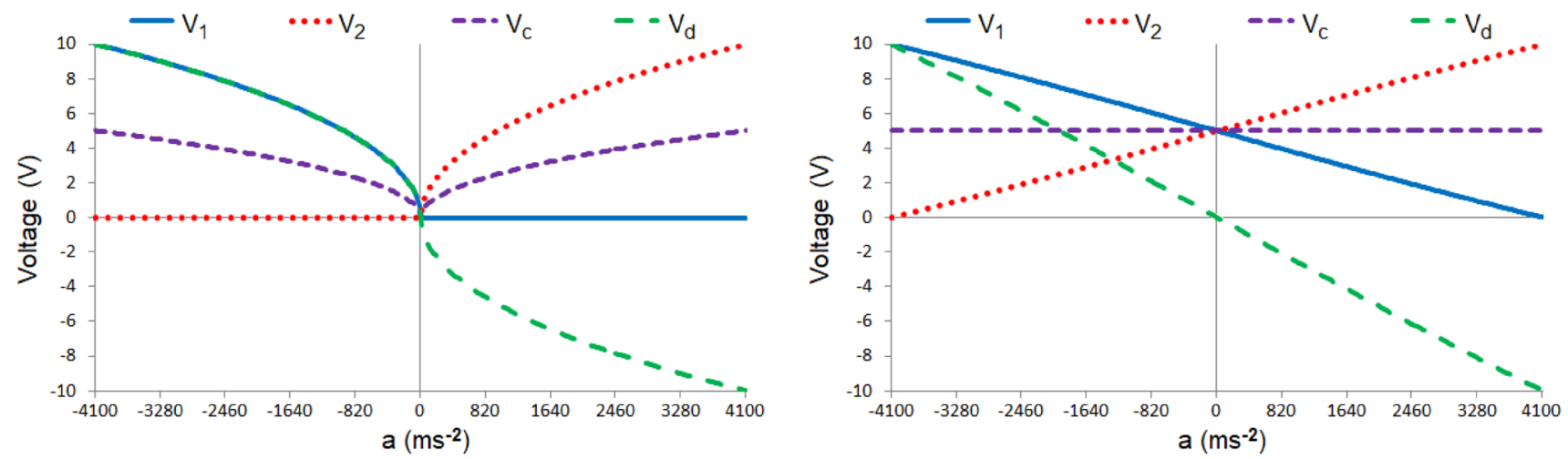

Fig. 23 Resting control voltages in the negative feedback regime versus acceleration based on FBCs. $m=50 \mathrm{ng}$ and $L_{s}=25 \mu \mathrm{m}$ 
and dynamic ranges are made possible due to the voltage control nature and cascaded architecture of the sensor.

Figure 24 displays the excursions and relative deformations of the four suspended Si beams of one section of the sensor for the length $L_{s}=25 \mu \mathrm{m}$ based on FBCs utilizing negative feedback control when applying $V_{1}=0$ and $V_{2}=10 \mathrm{~V}$ at an acceleration of $a=4100 \mathrm{~ms}^{-2}$ according to the characteristics of Fig. 23. Although the applied acceleration, in this case, is extremely high the difference voltage $V_{d}=-10 \mathrm{~V}$ maintains $\Delta \phi \approx 0$ and struggles to keep the seismic mass within still a reasonable distance of about $x=24.6 \mathrm{~nm}$ from the rest position. This corresponds to a mechanical sensitivity of about $0.0588 \mathrm{~nm} / \mathrm{g}$ of the seismic mass.

Although the studied sensor is not thermally actuated depending on the application the change in ambient temperature may be a concern. As indicated in Sect. 3 the temperature-dependent change in the effective index of the slot waveguide is minimal in the order of $1.2071 \times 10^{-4}$ $\left({ }^{\circ} \mathrm{F}\right)^{-1}$ based on the simulation results of VPIphotonics. However, the change in the thermoelastic properties of the suspended slot waveguides may affect the deformation of the beams due to the thermal stress. The $\mathrm{Si}$ is isotropic in thermal expansion owing to its diamond-like crystallographic structure. At the room temperature of $300 \mathrm{~K}$, the linear thermal expansion coefficient of $\mathrm{Si}$ is small in the order of $2.63 \times 10^{-6} \mathrm{~K}^{-1}$ [53]. Additionally, because the suspended Si beams are placed in the arms of a balanced MZI detection circuit and that the temperature change is going to be similar for all beams it is believed that the thermal effect would be neutralized being minimal in affecting the characteristics of the proposed sensor. The sensor characteristics simulated in this section including the spring constant, deflection of the seismic mass, modulated phase, and the mechanical and optical sensitivities are nonlinear functions of the common voltage in the open-loop control regime. However, the phase and voltage detection characteristics are almost purely linear functions of the measured acceleration. The programmable sensor may advantage smart software-based algorithms in the detection unit to switch between different operating regimes.

Table 2 summarizes the important design and simulation parameters for the cases of two and nine cascaded sections of the sensor taking $L_{s}=25 \mu \mathrm{m}$. As demonstrated in this investigation the value of $L_{s}=25 \mu \mathrm{m}$ have resulted in a moderate bandwidth response of the sensor and super optical sensitivities and dynamic ranges of acceleration measurement. Reducing the length $L_{s}$ of the suspended slot waveguides would have the effect of increasing the bandwidth response and although the optical sensitivity at reset voltages would decrease the shorter length of $L_{s}$ would allow using higher equal electrode voltages to increase the optical sensitivity to reasonably high values. The strength of the suspension beams against the weight of the seismic mass at the length $L_{s}=25 \mu \mathrm{m}$ is indicated in Table 2. The shift in the seismic mass due to the gravitational acceleration along the vertical $y$-axis is just $0.023 \mathrm{~nm}$ when the chip is horizontally mounted above ground. This displacement is a very small fraction of the suspension beam height of $300 \mathrm{~nm}$ and it would have minimal effect on the sensor performance. The bearable shifts in the seismic mass indicated in the table when the sensor is subjected to strong impacts of $10^{4} \mathrm{~ms}^{-2}$ along the $x$-axis and $y$-axis at reset voltages confirm that the suspension structure is sturdy in manufacturing and operation. It is revealed that the strong point in this study is the ability to engineer the proposed tough sensor to match a wide spectrum of applications providing operational levels of bandwidth, sensitivity, and dynamic ranges.
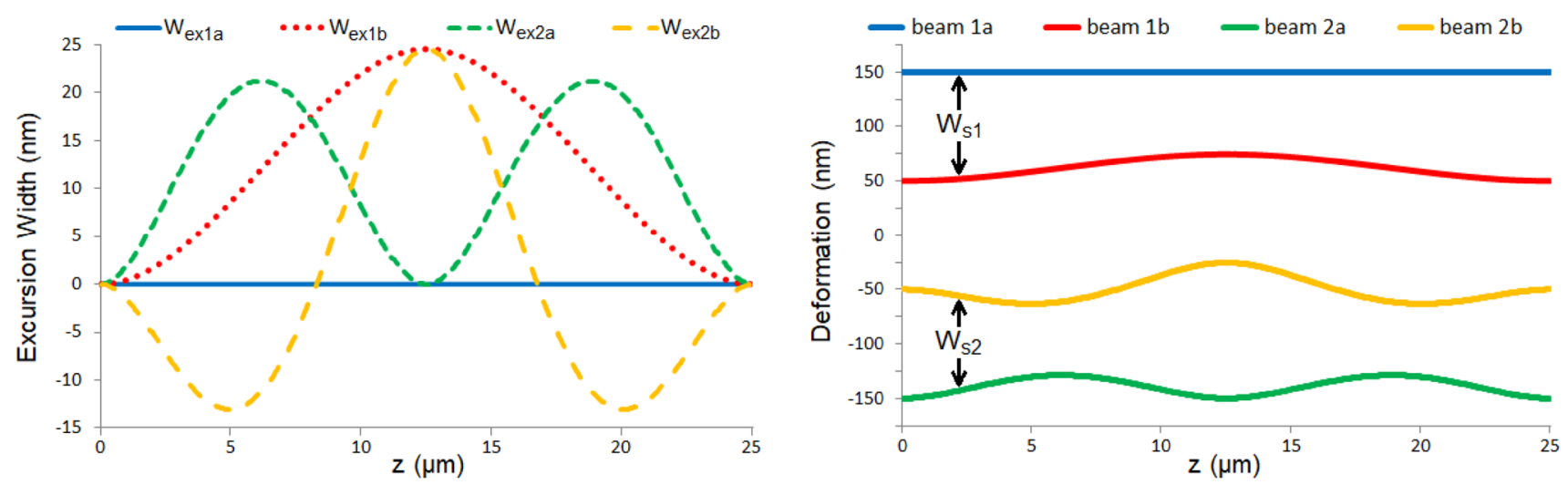

Fig. 24 Excursions of the four suspended Si beams based on FBCs. The right side part of the figure illustrates the relative deformations of the beams in the $x$-direction. $m=50 n g, L_{s}=25 \mu m, a=4100 \mathrm{~ms}^{-2}, V_{1}=0$, and $V_{2}=10 \mathrm{~V}$ 
Table 2 Summary of important design and simulation parameters

\begin{tabular}{|c|c|}
\hline Parameter & Value \\
\hline Si core layer thickness & $h=300 \mathrm{~nm}$ \\
\hline Box layer $\left(\mathrm{SiO}_{2}\right)$ thickness & $1-3 \mu \mathrm{m}$ \\
\hline Width of the Si beams of the slot waveguide & $W_{g s}=230 n m$ \\
\hline Slot width at reset voltages and zero acceleration & $W_{s 0}=100 n m$ \\
\hline Mass for one section of the seismic mass & $50 \mathrm{ng}$ \\
\hline Length of the suspended slot waveguide & $L_{s}=25 \mu \mathrm{m}$ \\
\hline Optical sensitivity & $\begin{array}{l}9.974-331.1 \% / \mathrm{g} \text { for two cascaded sections controlled by } V_{c} \text { at } V_{d}=0 \\
44.88-1489.8 \% / \mathrm{g} \text { for nine cascaded sections controlled by } V_{c} \text { at } V_{d}=0 \\
\text { Constant at } 0.239 \% / \mathrm{g} \text { in negative feedback control with } V_{c}=5 \mathrm{~V}\end{array}$ \\
\hline $\begin{array}{l}\text { Maximum measurable acceleration at full-scale deflection of the } \\
\text { seismic mass }\end{array}$ & $\begin{array}{l}98.32 \mathrm{~ms}^{-2} \text { for two cascaded sections at reset voltages } \\
21.85 \mathrm{~ms}^{-2} \text { for nine cascaded sections at reset voltages } \\
4100 \mathrm{~ms}^{-2} \text { in negative feedback control }\end{array}$ \\
\hline $\begin{array}{l}\text { Optical sensitivity dynamic range combining open-loop and closed- } \\
\text { loop operation }\end{array}$ & $\begin{array}{l}63 \mathrm{~dB} \text { for two cascaded sections } \\
76 \mathrm{~dB} \text { for nine cascaded sections }\end{array}$ \\
\hline Bandwidth response & Up to $25.2 \mathrm{kHz}$ at reset voltages \\
\hline $\begin{array}{l}\text { Shift in the seismic mass due to its weight along the lateral } x \text {-axis at } \\
\text { reset voltages when the chip is vertically mounted above ground }\end{array}$ & $0.39 \mathrm{~nm}$ \\
\hline $\begin{array}{l}\text { Shift in the seismic mass due to its weight along the vertical } y \text {-axis } \\
\text { at reset voltages when the chip is horizontally mounted above } \\
\text { ground }\end{array}$ & $0.023 \mathrm{~nm}$ \\
\hline $\begin{array}{l}\text { Shift in the seismic mass due to a strong impact of } 10^{4} \mathrm{~ms}^{-2} \text { along } \\
\text { the lateral } x \text {-axis at reset voltages }\end{array}$ & $40 \mathrm{~nm}$ \\
\hline $\begin{array}{l}\text { Shift in the seismic mass due to a strong impact of } 10^{4} \mathrm{~ms}^{-2} \text { along } \\
\text { the vertical } y \text {-axis at reset voltages }\end{array}$ & $23.4 \mathrm{~nm}$ \\
\hline
\end{tabular}

\section{Conclusions}

The SOI nanomachining with the under-etching fabrication step in the box layer is theoretically trialed for the construction of a photonic accelerometer made of seismic silicon mass suspended between the cascaded sections of two voltage-controlled slot waveguides that also form the two arms of an MZI photonic detection circuit of either broadband or periodic transmission response. The sensitivity of the proposed accelerometer is adaptive and can be controlled by electrode voltages. A total dynamic range of $76 \mathrm{~dB}$ of the optical sensitivity of the accelerometer combining both open-loop and closed-loop operation is proved in simulation. When the design of the suspension system of the accelerometer is optimized between high sensitivity and high operation speed the available detection bandwidth is shown possible in the $100 \mathrm{kHz}$ range for the seismic mass unit $m=50 \mathrm{ng}$. The characteristics of the accelerometer are studied including the phase difference, equivalent spring constant, deflection of the seismic mass, sensitivity dynamic range, and fundamental frequency of resonance of the suspension system. To inspect the deformation of the suspension beams based on the analysis presented in the research, the excursions of the four suspension beams used in one section of the accelerometer are shown for several examples of applying different accelerations and voltages. The deformation of the suspension beams in the future may also be verified utilizing a commercial simulation tool such as the COMSOL Multiphysics. The photonic sensor is shown to operate at low and high frequencies of the mechanical vibrations within the available bandwidth range. The application of negative feedback voltages to keep the seismic mass near a standstill is found advantageous to expand the measurable maximum acceleration way beyond the limit when no feedback is applied. Albeit the fact that the cascaded architecture of the proposed sensor allows increasing the bandwidth response in the sub-MHz range, the piezoelectric sensors that operate in the multiple $\mathrm{MHz}$ range better cover high operating speeds.

The dimensions of the components used in the core circuit of the photonic sensor are detailed should fabrication and testing of the device become accessible in the future. The lab testing allows addressing the fabrication challenges and possible device defects due to the sticky and adhesive mechanism of the movable parts and it allows evaluating the performance and characteristics of the accelerometer. In theory, it is found that the combined optical sensitivity of the device may electrostatically be controlled within an unprecedented range 
of $0.239-1489.8 \% / g$. However, the application of overrange electrode voltages may bring the suspension electromechanical system to its end limits of stability which also depends on the device orientation about the ground, and it may cause experiencing the pull-in effect and intensifying the Casimir-Lifshitz force between the movable parts when they get at close distances to each other on vibrations.

In future studies, it is also recommended to investigate the possibility to update the core processor architecture and detection system such that the device becomes capable of sensing accelerations in different orthogonal directions and sensing torsion forces. The accounting of the effects of air viscosity damping, squeeze-film air damping, and thermoelastic damping of the suspension beams and the effect of the optical gradient force in predicting the dynamic response of the device would stay challenging to future studies due to problem complexity. Add to that the current study considers the structure of the capacitance between the beams of the suspended slot waveguide to be ideal whereas it is not due to the limited heightto-width ratio of the beams. It is expected in the future that commercial simulation tools would make it possible to account for all these effects in sensors studied to fulfill the industrial and health care growing demands.

\section{Declarations}

Conflict of interest The author declares that he has no conflict of interest.

Ethical approval This article does not contain any studies with human participants or animals performed by the author.

Open Access This article is licensed under a Creative Commons Attribution 4.0 International License, which permits use, sharing, adaptation, distribution and reproduction in any medium or format, as long as you give appropriate credit to the original author(s) and the source, provide a link to the Creative Commons licence, and indicate if changes were made. The images or other third party material in this article are included in the article's Creative Commons licence, unless indicated otherwise in a credit line to the material. If material is not included in the article's Creative Commons licence and your intended use is not permitted by statutory regulation or exceeds the permitted use, you will need to obtain permission directly from the copyright holder. To view a copy of this licence, visit http://creativecommons. org/licenses/by/4.0/.

\section{References}

1. Acar C, Shkel AM (2003) Experimental evaluation and comparative analysis of commercial variable-capacitance MEMS accelerometers. J Micromech Microeng 13(5):634-645
2. Yuntao L, Xiaowei L, Weiping C, Qun W (2010) Design and noise analysis of a sigma-delta capacitive micromachined accelerometer. J Semicond 31(5):055006

3. Jiang $X$, Wang $F$, Kraft M, Boser BE(2002) An integrated surface micromachined capacitive lateral accelerometer with $2 \mu \mathrm{G} / \sqrt{ } \mathrm{Hz}$ resolution. Solid-state sensor, actuator and microsystems workshop, Hilton Head Island, South Carolina, 202-205

4. Wang YM, Chan PK, Li HKH, Ong SE (2015) A low-power highly sensitive capacitive accelerometer IC using autozero time-multiplexed differential technique. IEEE Sens J 15(11):6179-6191

5. Rani AD, Prasad PLHV, Prasad KR (2011) Design of a MEMS piezoelectric accelerometer. J Electr Eng 11(3):1-7

6. Kobayashi T, Okada H, Masuda T, Maeda R, Itoh T (2011) A digital output accelerometer using MEMS-based piezoelectric accelerometers and arrayed CMOS inverters with satellite capacitors. Smart Mater Struct 20(6):065017

7. Kasambe PV, Asgaonkar VV, Bangera AD, Lokre AS, Rathod SS, Bhoir DV (2018) Piezoelectric lead zirconate titanate (PZT) ring shaped contour-mode MEMS resonators. IOP Conf Series Mater Sci Eng 310(012069):1-10

8. Wang YH, Song P, Li X, Ru C, Ferrari G, Balasubramanian P, Amabili M, Sun Y, Liu X (2018) A paper-based piezoelectric accelerometer, MDPI. Micromachines 9(1):19

9. Noel W, Clerc PA, Dellmann L, Guldimann B, Herzig HP, Manzardo O, Marxer C, Weible K, Dändliker R, de Rooij N (2002) Applications of SOI-based optical MEMS. IEEE J Sel Top Quantum Electron 8(1):148-154

10. Zandi K, Wong B, Zou J, Kruzelecky RV, Jamroz W, Peter YA (2010) In-plane silicon-on-insulator optical MEMS accelerometer using waveguide Fabry-Perot microcavity with silicon/air Bragg mirrors. In: Proceedings of the IEEE international conference on Micro Electro Mechanical Systems (MEMS), pp 839-842

11. Westerveld WJ, Pozo J, Harmsma PJ, Schmits R, Tabak E, van den Dool TC, Leinders SM, van Dongen KWA, Urbach HP, Yousefi M (2012) Characterization of a photonic strain sensor in Si-oninsulator technology. Opt Lett 37(4):479-481

12. Krause AG, Winger M, Blasius TD, Lin Q, Painter O (2012) A microchip optomechanical accelerometer. Phy Opt 1-16

13. Zhao M, Jiang K, Bai $H$, Wang $H$, Wei X (2020) A MEMS based Fabry-Pe'rot accelerometer with high resolution, Springer Nature. Microsyst Technol 26:1961-1969

14. Malayappan B, Krishnaswamy N, Pattnaik PK (2020) Novel highresolution lateral dual-axis quad-beam optical MEMS accelerometer using waveguide Bragg gratings, MDPI. Photonics 7(3):49

15. Sheikhaleh A, Abedi K, Jafari K (2017) An optical MEMS accelerometer based on a two-dimensional photonic crystal add-drop filter. J Lightwave Technol 35(14):3029-3034

16. Sani MH, Saghaei H, Mehranpour MA, Tabrizi AA (2021) A novel all-optical sensor design based on a tunable resonant nanocavity in photonic crystal microstructure applicable in MEMS accelerometers. Photonic Sens 11(4):457-471

17. Miao H, Srinivasan K, Aksyuk V (2012) A microelectromechanically controlled cavity optomechanical sensing system. New J Phys 14:1-17

18. Guldimann B, Dubois P, Clerc PA, de Rooij N (2001) Fiber opticMEMS accelerometer with high mass displacement resolution. In: Proceedings of Transducers '01 Eurosensors XV. Springer, Berlin, Heidelberg, pp 438-441

19. Burcham KE, De Brabander GN, Boyd JT (1993) Micromachined silicon cantilever beam accelerometer incorporating an integrated optical waveguide. Proc SPIE Integr Optics Microstruct, USA 1793:12-18 
20. Malki A, Lecoy P, Marty J, Renouf C, Ferdinand P (1995) Optical fiber accelerometer based on a silicon micromachined cantilever. Appl Opt 34(34):8014-8018

21. Ahmadian M, Jafari K, Sharifi MJ (2018) Novel graphene-based optical MEMS accelerometer dependent on intensity modulation. ETRI J 40(6):794-801

22. Acoleyen KV, Roels J, Claes T, Thourhout DV, Baets R (2011) NEMS-based optical phase modulator fabricated on silicon-oninsulator. In: Proceedings of 8th IEEE international conference on Group IV Photonics (GFP), London, U.K, pp 371-373

23. Acoleyen KV, Roels J, Mechet P, Claes T, Thourhout DV, Baets $R$ (2012) Ultracompact phase modulator based on a cascade of NEMS-operated slot waveguides fabricated in silicon-on-insulator. IEEE Photonics J 4(3):779-788

24. Chiu WC, Chang CC, Wu JM, Lee MCM, Shieh JM (2011) Optical phase modulators using deformable waveguides actuated by micro-electro-mechanical systems. Opt Lett 36(7):1089-1091

25. Hussein AA, Majid SA, Hall TJ (2014) Design of compact tunable wavelength division multiplexing photonic phased array switches using nano-electromechanical systems on a silicon-on-insulator integration platform. Opt Quant Electron 46(10):1313-1320

26. Sheikhaleh A, Abedi K, Jafari K, Gholamzadeh R (2016) Microoptoelectromechanical systems accelerometer based on intensity modulation using a one-dimensional photonic crystal. Appl Opt 55(32):8993-8999

27. Zandi K, Zou J, Wong B, Kruzelecky RV, Peter YA (2011) VOAbased optical MEMS accelerometer. In: Proceedings of international conference on Optical MEMS and Nanophotonics (OMN), Istanbul, Turkey, pp 15-16

28. Zandi K, Bélanger JA, Peter YA (2012) Design and demonstration of an in-plane silicon-on-insulator optical MEMS Fabry-Pérotbased accelerometer integrated with channel waveguides. J Microelectromech Syst 21(6):1464-1470

29. Lorenzani S, Desvillettes L (2012) A kinetic approach for the evaluation of damping in micro-electro-mechanical systems devices vibrating at high frequencies. AIP Conf Proc Zaragoza Spain 1501(1):802-808

30. Lu Q, Fang W, Wang C, Bai J, Yao Y, Chen J, Xu X, Huang W (2021) Investigation of a complete squeeze-film damping model for MEMS devices. Microsyst Nanoeng 7(54):1-13

31. Kumar H, Mukhopadhyay S (2020) "Thermoelastic damping analysis in microbeam resonators based on Moore-Gibson-Thompson generalized thermoelasticity theory", Springer-Verlag GmbH Austria. Acta Mech 231:3003-3015

32. Almeida VR, Xu Q, Barrios CA, Lipson M (2004) Guiding and confining light in void nanostructure. Opt Lett 29(11):1209-1211

33. Lifshitz EM, Hamermesh M (1992) The theory of molecular attractive forces between solids. Perspectives in Theoretical Physics Elsevier 26:329-349

34. Lifshitz EM (1956) The theory of molecular attractive forces between solids. Soviet Physics 2(1):73-83

35. Povinelli ML, Lončar $M$, Ibanescu $M$, Smythe EJ, Johnson SG, Capasso F, Joannopoulos JD (2005) Evanescent-wave bonding between optical waveguides. Opt Lett 30(22):3042-3044

36. Povinelli $M L$, Johnson SG, Lončar $M$, Ibanescu $M$, Smythe EJ, Capasso F, Joannopoulos JD (2005) High-Q enhancement of attractive and repulsive optical forces between coupled whispering-gallery-mode resonators. OSA, Optics Express 13(20):8286-8295

37. Rodriguez AW, Hui PC, Woolf DN, Johnson SG, Loncar M, Capasso $F$ (2015) Classical and fluctuation-induced electromagnetic interactions in micronscale systems: designer bonding, antibonding, and Casimir forces. Ann Phys 527(1-2):45-80

38. De Leonardis F, De Carlo M, Passaro VMN (2017) Design rules for a nano-opto-mechanical actuator based on suspended slot Waveguides. MDPI, Photonics 4(43):1-8

39. Li M, Pernice WHP, Xiong C, Baehr-Jones T, Hochberg M, Tang HX (2008) Harnessing optical forces in integrated photonic circuits. Nature 456:480-484

40. Feng Y, Thomson DJ, Mashanovich GZ, Yan J (2020) Performance analysis of a silicon NOEMS device applied as an optical modulator based on a slot waveguide. Opt Express 28(25):38206-38222

41. Seifried $M$, Villares $G$, Baumgartner $Y$, Hahn $H$, Halter $M$, Horst F, Caimi D, Caër C, Sousa M, Dangel RF, Czornomaz L, Offrein BJ (2018) Monolithically integrated CMOS-compatible III-V on silicon lasers. IEEE J Sel Top Quantum Electron 24(6):1-9

42. Xiong C, Bell B, Eggleton BJ (2016) CMOS-compatible photonic devices for single-photon generation. Nanophotonics 5(3):427-439

43. Mauthe $S$, Triviño NV, Baumgartner Y, Sousa M, Caimi D, Stöferle T, Schmid H, Moselund KE (2019) InP-on-Si optically pumped microdisk lasers via monolithic growth and wafer bonding. IEEE J Sel Top Quantum Electron 25(6):1-7

44. Mu X, Wu S, Cheng L, Fu HY (2020) Edge couplers in silicon photonic integrated circuits: a review. MDPI, Appl Sci 10(4):1-29

45. Cheng L, Mao S, Li Z, Han Y, Fu HY (2020) Grating couplers on silicon photonics: design principles, emerging trends and practical issues. MDPI, Micromach 11(7):1-25

46. Sánchez-Postigo $A$, Halir R, Wangüemert-Pérez JG, OrtegaMoñux A, Wang S, Vachon M, Schmid JH, Xu DX, Cheben $P_{\text {, }}$ Molina-Fernández Í (2021) Breaking the coupling efficiencybandwidth trade-off in surface grating couplers using zeroorder radiation. Laser Photonics Rev 15(6):1-7

47. Sacher WD, Huang Y, Ding L, Barwicz T, Mikkelsen JC, Taylor BJF, Lo GQ, Poon JKS (2014) Polarization rotator-splitters and controllers in a Si3N4-on-SOI integrated photonics platform. Opt Express 22(9):11167-11174

48. Zhoua H, Zhaoa Y, Weia Y, Li F, Dong J, Zhang X (2019) All-inone silicon photonic polarization processor. Nanophotonics 8(12):2257-2267

49. Bachmann M, Besse PA, Melchior H (1994) General self-imaging properties in $\mathrm{N} \times \mathrm{N}$ multimode interference couplers including phase relations. Appl Opt 33(18):3905-3911

50. Soldano LB, Pennings ECM (1995) Optical multi-mode interference devices based on self-imaging: principles and applications. J Lightwave Technol 13(4):615-627

51. Hill MT, Leijtens XJM, Khoe GD, Smit MK (2003) Optimizing imbalance and loss in $2 \times 23-\mathrm{dB}$ multimode interference couplers via access waveguide width. J Lightwave Technol 21(10):2305-2313

52. Ye WN, Xu DX, Janz S, Cheben P, Picard MJ, Lamontagne B, Tarr NG (2005) Birefringence control using stress engineering in silicon-on-insulator (SOI) waveguides. J Lightwave Technol 23(3):1308-1318

53. Watanabe H, Yamada N, Okaji M (2004) Linear thermal expansion coefficient of silicon from 293 to $1000 \mathrm{~K}$. Int J Thermophys 25(1):221-236

Publisher's Note Springer Nature remains neutral with regard to jurisdictional claims in published maps and institutional affiliations. 